\title{
NEGATIVE CURVATURE IN GRAPHICAL SMALL CANCELLATION GROUPS
}

\author{
GOULNARA N. ARZHANTSEVA, CHRISTOPHER H. CASHEN, DOMINIK GRUBER, \\ AND DAVID HUME
}

\begin{abstract}
We use the interplay between combinatorial and coarse geometric versions of negative curvature to investigate the geometry of infinitely presented graphical $G r^{\prime}(1 / 6)$ small cancellation groups. In particular, we characterize their 'contracting geodesics', which should be thought of as the geodesics that behave hyperbolically.

We show that every degree of contraction can be achieved by a geodesic in a finitely generated group. We construct the first example of a finitely generated group $G$ containing an element $g$ that is strongly contracting with respect to one finite generating set of $G$ and not strongly contracting with respect to another. In the case of classical $C^{\prime}(1 / 6)$ small cancellation groups we give complete characterizations of geodesics that are Morse and that are strongly contracting.

We show that many graphical $G r^{\prime}(1 / 6)$ small cancellation groups contain strongly contracting elements and, in particular, are growth tight. We construct uncountably many quasi-isometry classes of finitely generated, torsion-free groups in which every maximal cyclic subgroup is hyperbolically embedded. These are the first examples of this kind that are not subgroups of hyperbolic groups.

In the course of our analysis we show that if the defining graph of a graphical $G r^{\prime}(1 / 6)$ small cancellation group has finite components, then the elements of the group have translation lengths that are rational and bounded away from zero.
\end{abstract}

\section{INTRODUCTION}

Graphical small cancellation theory was introduced by Gromov as a powerful tool for constructing finitely generated groups with desired geometric and analytic properties [26]. Its key feature is that it produces infinite groups with prescribed subgraphs in their Cayley graphs. The group properties are thus derived from the combinatorial or asymptotic properties of the embedded subgraphs. Over the last two decades, graphical small cancellation theory has become an increasingly prominent and versatile tool of geometric group theory with a wide range of striking examples and applications.

In this paper, we provide a thorough investigation of the hyperbolic-like geometry of graphical small cancellation constructions. Our theorems show that the constructed groups behave strongly like groups hyperbolic relative to their defining graphs. This contrasts the fact that in general they need not be Gromov hyperbolic or even relatively hyperbolic. With this geometric analogy in mind, we produce a variety of concrete examples and determine the spectrum of negative curvature possible in the realm of finitely generated groups.

2010 Mathematics Subject Classification. Primary: 20F06; Secondary: 20F65, $20 \mathrm{~F} 67$.

Key words and phrases. Graphical small cancellation, Morse quasi-geodesic, contracting projection, HEC property.

This work is supported by the ERC grant of Goulnara Arzhantseva "ANALYTIC" no. 259527. The second author is supported by the Austrian Science Fund (FWF):M1717-N25. The third author is supported by the Swiss National Science Foundation Professorship FN PP00P2-144681/1 of Laura Ciobanu. The fourth author is supported by the ERC grant no. 278469 of Pierre-Emmanuel Caprace and the grant ANR-14-CE25-0004 "GAMME" of Damien Gaboriau and Romain Tessera. 
Graphical small cancellation theory was first used by Gromov [26] in the description of the groups now known as 'Gromov monsters', which are finitely generated groups that contain sequences of expander graphs in their Cayley graphs. These monster groups do not coarsely embed into a Hilbert space, whence they are not coarsely amenable (i.e. do not have Yu's property A), and they give counterexamples to the Baum-Connes conjecture with coefficients [32]. Graphical small cancellation theory is currently the only means of proving the existence of finitely generated groups with any of these three properties.

Since Gromov's initial impetus, the theory has gained in significance and variety of applications. Indeed, the construction is very versatile: every countable group embeds into a 2-generated graphical small cancellation group [29, Example 1.13], and graphical small cancellation theory has been used, for instance, to give many new groups without the unique product property $[5,47,30]$, the first examples of non-coarsely amenable groups with the Haagerup property [4, 40], and new hyperbolic groups with Kazhdan's Property (T) $[26,44,39]$, as well as to build a continuum of Gromov monsters [33], the first examples of finitely generated groups that do not coarsely embed into Hilbert space and yet do not contain a weakly embedded expander [6], and to analyze the Wirtinger presentations of prime alternating link groups [18]. Moreover, since the class of graphical small cancellation groups contains all classical small cancellation groups, it contains, for example, groups having no finite quotients [42], groups with prescribed asymptotic cones [51, 22], and groups with exceptional divergence functions [31].

Our paper has two purposes. The first purpose is to study geometric aspects of graphical small cancellation groups. An important property we focus on is the existence of subspaces that 'behave like' subspaces of a negatively curved space. We quantify this phenomenon by considering contraction properties of a subspace. Intuitively, this measures the asymptotic growth of closest point projections of metric balls to the subspace. In a recent work [7], we defined a general quantitative spectrum of contraction in arbitrary geodesic metric spaces and used it to produce new results on the interplay between contraction, divergence, and the property of being Morse. Our definitions generalize prior notions of contraction that have been instrumental in the study of numerous examples of finitely generated groups of current interest, such as mapping class groups [35, 12, 23], outer automorphism groups of free groups [37, 2], and, more generally, acylindrically hyperbolic groups [19]. In the present paper, we completely determine the contraction properties of geodesics in graphical small cancellation groups through their defining graphs. En route, we describe geodesic polygons and translation lengths in these groups.

The second purpose of this paper is to detect the range of possible contracting behaviors in finitely generated groups. To this end, we use graphical small cancellation theory to show that every degree of contraction can be achieved by a geodesic in a suitable group. Moreover, we give the first examples of strongly contracting geodesics that are not preserved under quasi-isometries of groups. These results further establish the graphical small cancellation technique as a fundamental source of novel examples of finitely generated groups.

Our main technical result is a local-to-global theorem for the contraction properties of geodesics in graphical small cancellation groups. It states that the contraction function of a geodesic is measured by its intersections with the defining graph. This confirms the analogy with relatively hyperbolic spaces and their peripheral subspaces. Beyond the applications alluded to above, the theorem also enables us to prove the general result that many infinitely presented graphical small cancellation groups contain strongly contracting elements and, in particular, are growth tight, and to provide a characterization of Morse geodesics in classical $C^{\prime}(1 / 6)$ small cancellation groups. Furthermore, using the 
fact that strongly contracting elements give rise to hyperbolically embedded virtually cyclic subgroups, we produce the first examples of torsion-free groups in which every element is contained in a maximal virtually cyclic hyperbolically embedded subgroup but that are not subgroups of hyperbolic groups.

The proof of our local-to-global theorem rests on a meticulous analysis of the geometry of the Cayley graphs of graphical $G r^{\prime}(1 / 6)$ small cancellation groups. In particular, we provide a complete classification of the geodesic quadrangles in the Cayley graphs of these groups, which is of independent interest and is new even for classical $C^{\prime}(1 / 6)$-groups.

The general tools that we establish have additional applications. For instance, we show that in many infinitely presented graphical small cancellation groups, the translation lengths of infinite order elements are rational and bounded away from zero. These tools will undoubtedly be useful towards further applications of this very interesting class of groups.

In the remainder of this introduction we explain the key concepts and main results of this paper in more detail, and give a brief overview of the proof of our local-to-global theorem.

Acknowledgements. A part of this work was developed during the program Measured group theory held at the Erwin Schrödinger Institute for Mathematics and Physics in Vienna in 2016. We thank its organizers and the institute for its hospitality. We also thank the Isaac Newton Institute for Mathematical Sciences for support and hospitality during the program Non-positive curvature: group actions and cohomology. This work is partially supported by EPSRC Grant Number EP/K032208/1.

Finally, we thank the referees for their careful reading of the paper and their helpful comments.

1.1. Contracting subspaces. In the following we shall assume that $X$ is a geodesic metric space such that for every closed $Y \subset X$ and $x \in X$, the set $\pi(x):=\{y \in Y \mid$ $d(x, y)=d(x, Y)\}$ is non-empty. This is true for a proper space $X$, but also for a connected graph (i.e. a connected 1-dimensional CW-complex) $X$. We call $\pi$ closest point projection to $Y$. We do not assume the sets $\pi(x)$ have uniformly bounded diameter.

Definition 1.1 (Contracting). Let $Y$ be a closed subspace of $X$, and denote by $\pi$ the closest point projection to $Y$. Let $\rho_{1}$ and $\rho_{2}$ be non-decreasing, eventually non-negative functions, with $\rho_{1}(r) \leqslant r$ and $\rho_{1}$ unbounded. We say that $Y$ is $\left(\rho_{1}, \rho_{2}\right)$-contracting if the following conditions are satisfied for all $x, x^{\prime} \in X$ :

- $d\left(x, x^{\prime}\right) \leqslant \rho_{1}(d(x, Y)) \Longrightarrow \operatorname{diam} \pi(x) \cup \pi\left(x^{\prime}\right) \leqslant \rho_{2}(d(x, Y))$

- $\lim _{r \rightarrow \infty} \frac{\rho_{2}(r)}{\rho_{1}(r)}=0$

If $\rho_{1}(r)=r$, then we say $Y$ is sublinearly contracting, if $\rho_{1}(r)=r$ and $\rho_{2}(r)=C$ for some constant $C$ we say it is strongly contracting, and if $\rho_{1}(r)=r / 2$ and $\rho_{2}(r)=C$ for some constant $C$ we say it is semi-strongly contracting

We say a function $f$ is sublinear if it is non-decreasing, eventually non-negative, and $\lim _{r \rightarrow \infty} \frac{f(r)}{r}=0$.

The most basic example of a strongly contracting subspace is a geodesic in a tree or, more generally, a geodesic in a $\delta$-hyperbolic space. The opposite extreme occurs in a Euclidean space where there are no contracting geodesics (for any choice of $\rho_{1}$ and $\rho_{2}$ ). The contrast between hyperbolic and Euclidean type behavior is evident in the following well-known examples of contraction: 
- A geodesic in a CAT(0) space is strongly contracting if and only if it is Morse $[14,49]$.

- A geodesic in a relatively hyperbolic space is strongly contracting if for every $C \geqslant 0$ there exists a $B \geqslant 0$ such that the geodesic spends at most time $B$ in the $C$-neighborhood of a peripheral subset [45].

The common idea in these situations is that the given space has certain regions that are not hyperbolic, but geodesics that avoid these non-hyperbolic regions behave very much like geodesics in a hyperbolic space. Similar phenomena occur for pseudo-Anosov axes in the Teichmüller space of a hyperbolic surface and iwip axes in the Outer Space of the outer automorphism group of a free group. Such axes avoid the 'thin parts' of their respective spaces and therefore are strongly contracting [37, 2].

A version of semi-strong contraction, where the projection is not necessarily closest point projection, occurs for pseudo-Anosov axes in the mapping class group of a hyperbolic surface $[35,12,23]$.

1.2. Local-to-global theorem. Given a directed graph $\Gamma$ whose edges are labelled by the elements of a set $\mathcal{S}$, the group defined by $\Gamma$, denoted $G(\Gamma)$ is given by the presentation $\langle\mathcal{S}|$ labels of embedded cycles in $\Gamma\rangle$. The graphical $G r^{\prime}(1 / 6)$ small cancellation condition, see Section 2.1, is a combinatorial requirement on the labelling of $\Gamma$, whose key consequence is that the connected components of $\Gamma$ isometrically embed into $\operatorname{Cay}(G(\Gamma), \mathcal{S})$. In the case that $\Gamma$ is a disjoint union of cycle graphs labelled by a set of words $\mathcal{R}$, the graphical $G r^{\prime}(1 / 6)$-condition for $\Gamma$ corresponds to the classical $C^{\prime}(1 / 6)$-condition for $\mathcal{R}$.

We show that, similar to the situations described above, geodesics in Cayley graphs of graphical $G r^{\prime}(1 / 6)$ small cancellation groups behave like hyperbolic geodesics as long as they avoid the embedded components of the defining graph. In fact, a geodesic is as hyperbolic as its intersections with the embedded components of $\Gamma$ :

Theorem (Theorem 4.1). Let $\Gamma$ be a $G r^{\prime}(1 / 6)$-labelled graph. There exist $\rho_{1}^{\prime}$ and $\rho_{2}^{\prime}$ such that a geodesic $\alpha$ in $X:=\operatorname{Cay}(G(\Gamma), \mathcal{S})$ is $\left(\rho_{1}^{\prime}, \rho_{2}^{\prime}\right)$-contracting if and only if there exist $\rho_{1}$ and $\rho_{2}$ such that for every embedded component $\Gamma_{0}$ of $\Gamma$ in $X$ such that $\Gamma_{0} \cap \alpha \neq \emptyset$, we have that $\Gamma_{0} \cap \alpha$ is $\left(\rho_{1}, \rho_{2}\right)$-contracting as a subspace of $\Gamma_{0}$.

Moreover, $\rho_{1}^{\prime}$ and $\rho_{2}^{\prime}$ can be bounded in terms of $\rho_{1}$ and $\rho_{2}$, and when $\rho_{1}(r) \geqslant r / 2$ we can take $\rho_{1}^{\prime}=\rho_{1}$ and $\rho_{2}^{\prime} \asymp \rho_{2}$.

Here $\asymp$ denotes a standard notion of asymptotic equivalence, see Section 2. Our theorem gives the following explicit application to classical $C^{\prime}(1 / 6)$-groups. We denote by $|\cdot|$ the number of edges of a path graph or cycle graph.

Theorem (Corollary 4.14). Let $\Gamma$ be a $G r^{\prime}(1 / 6)$-labelled graph whose components are cycle graphs. Let $\alpha$ be a geodesic in $X:=\operatorname{Cay}(G(\Gamma), \mathcal{S})$. Define $\rho(r):=\max _{\left|\Gamma_{i}\right| \leqslant r}\left|\Gamma_{i} \cap \alpha\right|$, where the $\Gamma_{i}$ range over embedded components of $\Gamma$ in $X$. Then $\alpha$ is sublinearly contracting if and only if $\rho$ is sublinear, in which case $\alpha$ is $(r, \rho)$-contracting. In particular, $\alpha$ is strongly contracting if and only if $\rho$ is bounded.

1.3. Morse geodesics. A classically more well-studied notion of what it means to behave like a subspace of a hyperbolic space is the property of being Morse.

Definition 1.2 (Morse). A subspace $Y$ of a geodesic metric space $X$ is $\mu$-Morse if every $(L, A)$-quasi-geodesic in $X$ with endpoints on $Y$ is contained in the $\mu(L, A)$-neighborhood of $Y$. A subspace is Morse if there exists some $\mu$ such that it is $\mu$-Morse.

The property of being Morse is invariant under quasi-isometries, and the fact that quasi-geodesics in a Gromov hyperbolic space are Morse is known as the 'Morse Lemma'. 
These two results are main ingredients in the proof that hyperbolicity is preserved by quasi-isometries. Morse geodesics are of further interest due to their close connection with the geometry of asymptotic cones and relations with other important geometric concepts such as divergence, see for example $[21,11,7]$ and references therein. In [7], we prove that being contracting is, in fact, equivalent to being Morse.

Theorem 1.3 ([7, Theorem 1.4]). If $Y$ is a subspace of a geodesic metric space such that the empty set is not in the image of closest point projection to $Y$, then $Y$ is Morse if and only if $Y$ is $\left(\rho_{1}, \rho_{2}\right)$-contracting for some $\rho_{1}$ and $\rho_{2}$.

Thus, in the case of classical $C^{\prime}(1 / 6)$-groups, Theorem 4.1 and Theorem 1.3 enable us to provide a complete characterization of Morse geodesics in the Cayley graph.

Theorem (Corollary 4.14). Let $\Gamma$ be a $G r^{\prime}(1 / 6)$-labelled graph whose components are cycle graphs. Let $\alpha$ be a geodesic in $\operatorname{Cay}(G(\Gamma), \mathcal{S})$. Define $\rho(r):=\max _{\left|\Gamma_{i}\right| \leqslant r}\left|\Gamma_{i} \cap \alpha\right|$, where the $\Gamma_{i}$ range over embedded components of $\Gamma$. Then $\alpha$ is Morse if and only if $\rho$ is sublinear.

1.4. Range of contracting behaviors. As mentioned, in a $\operatorname{CAT}(0)$-space, a geodesic is Morse if and only if it is strongly contracting. Thus, Theorem 1.3 says that in a $\operatorname{CAT}(0)$ space a geodesic is either strongly contracting or not contracting at all. We show that in finitely generated groups, the spectrum of contraction is, in fact, much richer: every degree of contraction can be attained.

Theorem (Theorem 4.15). Let $\rho$ be a sublinear function. There exists a group $G$ with finite generating set $\mathcal{S}$ and a sublinear function $\rho^{\prime} \asymp \rho$ such that there exists an $\left(r, \rho^{\prime}\right)$-contracting geodesic $\alpha$ in $\operatorname{Cay}(G, \mathcal{S})$, and $\rho^{\prime}$ is optimal, in the sense that if $\alpha$ is $\left(r, \rho^{\prime \prime}\right)$-contracting for some other function $\rho^{\prime \prime}$ then $\lim \sup _{r \rightarrow \infty} \frac{\rho^{\prime \prime}(2 r)}{\rho(r)} \geqslant 1$.

Furthermore $\alpha$ can be chosen to be within finite Hausdorff distance of a cyclic subgroup of $G$.

Our current examples are not finitely presentable. The contraction spectrum for finitely presented groups remains largely unexplored. Indeed, if one restricts to geodesics within finite Hausdorff distance of a cyclic subgroup, finitely presented groups can only display countably many degrees of contraction.

Question 1.4. For which functions $\rho$ do there exist finitely presented groups $G$ containing a geodesic in some Cayley graph that is $(r, \rho)$-contracting?

1.5. Non-stability of strong contraction. While the property of being Morse is stable under quasi-isometries, it has remained unknown whether the property of being strongly contracting is. We provide a negative answer by providing the first examples of spaces $X$ and $\tilde{X}$ and geodesics $\gamma$ and $\tilde{\gamma}$ such that there exists a quasi-isometry $X \rightarrow \tilde{X}$ mapping $\gamma$ to $\tilde{\gamma}$ and such that $\gamma$ is not strongly contracting, but $\tilde{\gamma}$ is strongly contracting.

Theorem (Theorem 4.19). There exists a group $G$ with finite generating sets $\mathcal{S} \subset \tilde{\mathcal{S}}$ and an infinite geodesic $\gamma$ in $X:=\operatorname{Cay}(G, \mathcal{S})$ labelled by the powers of a generator such that $\gamma$ is not strongly contracting, but its image $\tilde{\gamma}$ in $\tilde{X}:=\operatorname{Cay}(G, \tilde{\mathcal{S}})$ obtained from the inclusion $\mathcal{S} \subset \tilde{\mathcal{S}}$ is an infinite strongly contracting geodesic.

Indeed, in many familiar settings, such as hyperbolic groups, CAT(0) groups, or toral relatively hyperbolic groups, such examples could not be obtained, since in those contexts, strong contraction is equivalent to the Morse property. 
1.6. Strongly contracting elements and growth tightness. Another of our main results is the existence of strongly contracting elements in many graphical small cancellation groups:

Theorem (Theorem 5.1). Let $\Gamma$ be a $G r^{\prime}(1 / 6)$-labelled graph whose components are finite, labelled by a finite set $\mathcal{S}$. Assume that $G(\Gamma)$ is infinite. Then there exists an infinite order element $g \in G(\Gamma)$ such that $\langle g\rangle$ is strongly contracting in $\operatorname{Cay}(G(\Gamma), \mathcal{S})$.

The element $g$ is, in fact, the WPD element for the action on the hyperbolic conedoff space in Gruber and Sisto's proof of acylindrical hyperbolicity of these groups [31]. Theorem 5.1 has the following consequence (which does not follow from acylindrical hyperbolicity):

Arzhantseva, Cashen, and Tao [8] have shown that the action of a finitely generated group $G$ on a Cayley graph $X$ is growth tight if the action has a strongly contracting element, that is, an element $g$ such that $\langle g\rangle$ is strongly contracting in $X$. Growth tightness means that the exponential growth rate of an orbit of $G$ in $X$ is strictly greater than the growth rate of an orbit of $G / N$ in $N \backslash X$, for every infinite normal subgroup $N$. Theorem 5.1 therefore implies:

Theorem (Theorem 5.2). Let $\Gamma$ be a $G r^{\prime}(1 / 6)$-labelled graph whose components are finite, labelled by a finite set $\mathcal{S}$. Then the action of $G(\Gamma)$ on $\operatorname{Cay}(G(\Gamma), \mathcal{S})$ is growth tight.

This has raised our interest in the following question, first asked by Grigorchuk and de la Harpe for hyperbolic fundamental groups of closed orientable surfaces [24]:

Question 1.5. Let $\Gamma$ be a $G r^{\prime}(1 / 6)$-labelled graph whose components are finite, labelled by a finite set $\mathcal{S}$. Does $G(\Gamma)$ attain its infimal growth rate with respect to the generating set $\mathcal{S}$ ?

Together with Theorem 5.2, a positive answer, even for the subclass of classical $C^{\prime}(1 / 6)$ groups, would establish small cancellation theory as an abundant source of Hopfian groups.

1.7. Contraction and hyperbolically embedded subgroups. An important application of the notion of strong contraction is the fact that an infinite order element whose orbit in the Cayley graph is strongly contracting is contained in a virtually cyclic hyperbolically embedded subgroup [19], see Definition 6.1. In particular, our proof of Theorem 5.1 gives a new argument that the WPD elements of [31] produce hyperbolically embedded subgroups. Furthermore, our methods detect strongly contracting elements that need not be hyperbolic elements for the action on the coned-off space of [31], see Example 4.18 and Remark 5.8. Admitting a proper infinite hyperbolically embedded subgroup is equivalent to being acylindrially hyperbolic [41], which implies a number of strong group theoretic properties.

Not all hyperbolically embedded virtually cyclic subgroups are strongly contracting; see [8] for an example. However, every hyperbolically embedded subgroup of a finitely generated group is Morse [46]. In light of Theorem 1.3, a natural question is whether there exists some critical rate of contraction that guarantees a subgroup is hyperbolically embedded. That is, does there exist an unbounded sublinear function $\rho_{2}$ such that every element $g$ with a $\left(r, \rho_{2}\right)$-contracting orbit in some Cayley graph has a hyperbolically embedded virtually cyclic elementary closure? The elementary closure of $g$ is the subgroup generated by all virtually cyclic subgroups containing $g$. We prove no such $\rho_{2}$ exists.

Theorem (Theorem 6.4). Let $\rho_{2}$ be an unbounded sublinear function. There exists a $G r^{\prime}(1 / 6)$-labelled graph $\Gamma$ with set of labels $\mathcal{S}:=\{a, b\}$ whose components are all cycles such 
that $G(\Gamma)$ has the following properties: Any virtually cyclic subgroup $E$ of $G(\Gamma)$ containing $\langle a\rangle$ is $\left(r, \rho_{2}^{\prime}\right)$-contracting in the Cayley graph $\operatorname{Cay}(G(\Gamma), \mathcal{S})$ for some $\rho_{2}^{\prime} \asymp \rho_{2}$, but $E$ is not hyperbolically embedded in $G(\Gamma)$.

1.8. Hyperbolically embedded cycles. In a subgroup of a hyperbolic group, every infinite order element is contained in a maximal virtually cyclic, hyperbolically embedded subgroup, whence we define the following:

Definition 1.6 (HEC property). A group has the hyperbolically embedded cycles property (HEC property) if the elementary closure $E(g)$ of every infinite order element $g$ is virtually cyclic and hyperbolically embedded.

It is natural to ask whether this property characterizes subgroups of hyperbolic groups. While torsion presents an obvious complication, see Section 6.2, we also present a negative answer to our question in the torsion-free case.

Theorem (Theorem 6.6). There exist $2^{\aleph_{0}}$ pairwise non-quasi-isometric finitely generated torsion-free groups in which every non-trivial cyclic subgroup is strongly contracting and which, therefore, have the HEC property.

These are the first examples of groups of this kind that do not arise as subgroups of hyperbolic groups. Our examples include exotic specimens such as Gromov monsters.

1.9. Translation lengths. Let $|\cdot|$ be the word length in $G(\Gamma)$ with respect to $\mathcal{S}$. The translation length of an element $g \in G(\Gamma)$ is:

$$
\tau(g):=\lim _{n \rightarrow \infty} \frac{\left|g^{n}\right|}{n}
$$

Conner [16] calls a group whose non-torsion elements have translation length bounded away from zero translation discrete. Hyperbolic groups [50], CAT(0) groups [17], and finitely presented groups satisfying various classical small cancellation conditions [34] are translation discrete.

We show that many (possibly infinitely presented) graphical small cancellation groups are also translation discrete:

Theorem (Theorem 5.4). Let $\Gamma$ be a $G r^{\prime}(1 / 6)$-labelled graph whose components are finite, labelled by a finite set $\mathcal{S}$. Then every infinite order element of $G(\Gamma)$ has rational translation length, and translation lengths are bounded away from zero.

1.10. The idea of the proof of the local-to-global theorem. In a tree, geodesic quadrangles are degenerate, as seen in Figure 1. In a hyperbolic space, geodesic quadrangles
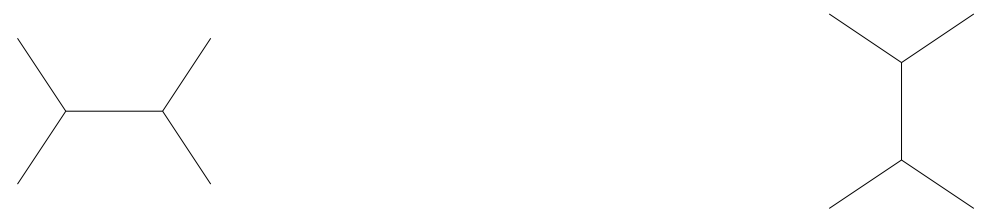

Figure 1. Tree quadrangles

can be approximated by geodesic quadrangles in a tree. If the base is a fixed geodesic $\alpha$, the top is some given geodesic $\gamma$, and the sides are given by closest point projection from the endpoints of the top to the bottom, then the resulting geodesic quadrangle is either 'short' or 'thin', as in Figure 2.

In Proposition 3.20, we show a combinatorial version of this dichotomy through an analysis of van Kampen diagrams in graphical $G r^{\prime}(1 / 6)$ small cancellation groups. Specifically, 

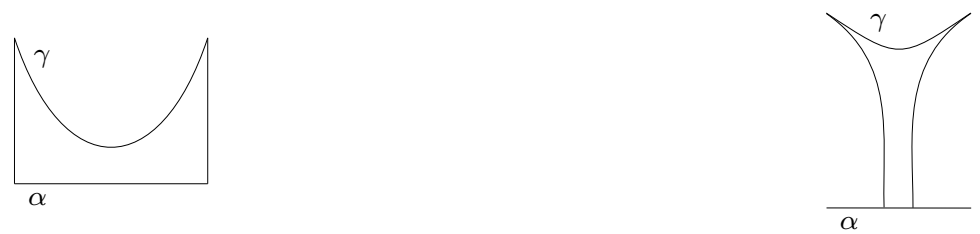

FiguRE 2. Hyperbolic quadrangles from closest point projection

if $X:=\operatorname{Cay}(G(\Gamma), \mathcal{S})$ and $\alpha \subset X$ is a $\left(\rho_{1}, \rho_{2}\right)$-contracting geodesic, $\gamma \subset X$ is another geodesic, and each endpoint of $\gamma$ is connected via a geodesic to a closest point of $\alpha$, then the boundary word of the resulting geodesic quadrangle admits a van Kampen diagram that is either 'short' or 'thin' in terms of number of faces, as depicted in Figure 3.
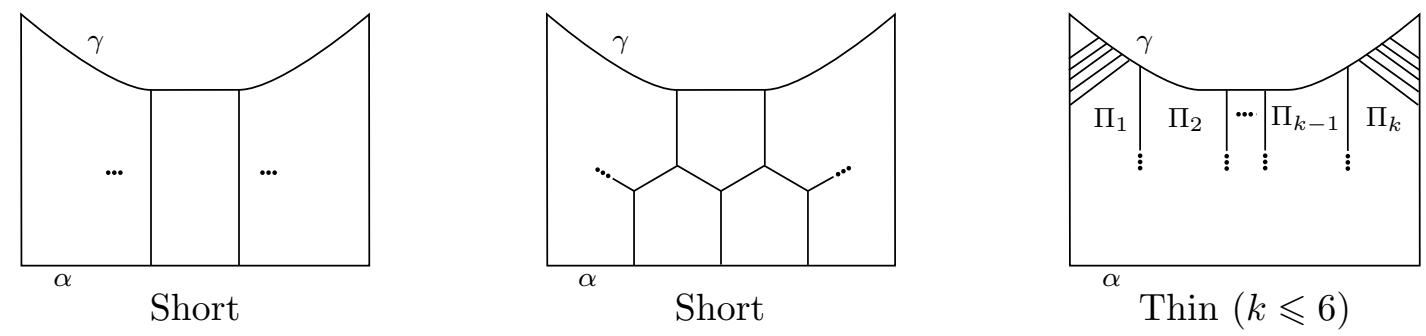

Figure 3. Combinatorially short and thin quadrangles

The main ingredient in establishing this dichotomy is a classification of 'special combinatorial geodesic quadrangles', see Theorem 3.18, that extends Strebel's classification of geodesic bigons and triangles in small cancellation groups, see Theorem 3.13. This classification is of independent interest, and is novel even within the class of classical small cancellation groups.

These combinatorial versions of short and thin quadrangles do not immediately imply their metric counterparts, because the faces in the van Kampen diagrams may have boundary words that are arbitrarily long relators. In Section 4 we use the fact that $\alpha$ is $\left(\rho_{1}, \rho_{2}\right)$-contracting to show that even if the faces have long boundaries, their projections to $\alpha$ are small with respect to their distance from $\alpha$.

The essential trick that is used repeatedly is to play off the small cancellation condition against the contraction condition. Specifically, if $\Pi$ is a face of the van Kampen diagram with few sides, one of which sits on $\alpha$, we use the small cancellation condition to show that $|\alpha \cap \partial \Pi|$ is bounded below by a linear function of $|\partial \Pi|$. Then we use the contraction condition to say that $|\alpha \cap \partial \Pi|$ is bounded above by a sublinear function of $|\partial \Pi|$. Thus, we have a sublinear function of $|\partial \Pi|$ that gives an upper bound to a linear function of $|\partial \Pi|$. This is only possible if $|\partial \Pi|$ is smaller than some bound depending on the two functions.

\section{Preliminaries}

We set notation. Let $\mathcal{S}$ be a set.

- $\langle\mathcal{S}\rangle$ is the group generated by $\mathcal{S}$. If $\mathcal{S}$ is a subset of a group $G$ then $\langle\mathcal{S}\rangle$ is the subgroup of $G$ generated by $\mathcal{S}$. If $\mathcal{S}$ is a set of formal symbols then $\langle\mathcal{S}\rangle$ is the free group freely generated by $\mathcal{S}$.

- If $\mathcal{S}$ is a set of formal symbols then $\mathcal{S}^{-}:=\left\{s^{-1} \mid s \in \mathcal{S}\right\}$ is the set of formal inverses, and $\mathcal{S}^{ \pm}:=\mathcal{S} \cup \mathcal{S}^{-}$.

- $\mathcal{S}^{*}$ is the free monoid over $\mathcal{S}$.

- $2^{\mathcal{S}}$ is the set of subsets of $\mathcal{S}$.

- $\mathcal{S}^{\mathbb{N}}$ is the set of infinite sequences with terms in $\mathcal{S}$. 
We write $f \preceq g$ if there exist $C_{1}>0, C_{2}>0, C_{3} \geqslant 0$, and $C_{4} \geqslant 0$ such that $f(x) \leqslant C_{1} g\left(C_{2} x+C_{3}\right)+C_{4}$ for all $x$. If $f \preceq g$ and $g \preceq f$ then we write $f \asymp g$.

Note that if $f \preceq g$ and $g$ is bounded then $f$ is bounded, and if $f$ is non-decreasing and eventually non-negative and $f \preceq g$ for a function $g$ such that $\lim _{r \rightarrow \infty} \frac{g(r)}{r}=0$, then $f$ is sublinear.

The girth of a graph is the length of its shortest non-trivial cycle.

2.1. Graphical small cancellation. Graphical small cancellation theory is a generalization of classical small cancellation theory. The main application is an embedding of a desired sequence of graphs into the Cayley graph of a group. It was introduced by Gromov [26], and was later clarified and expanded by Ollivier [38], Arzhantseva and Delzant [9], and, in a systematic way, by Gruber [27].

2.1.1. Basic facts. Let $\Gamma$ be a directed graph with edges labelled by a set $\mathcal{S}$. We allow paths to traverse edges against their given direction, with the convention that the label of an oppositely traversed edge is the formal inverse of the given label. Thus, given a finite path in $\Gamma$ we can read a word in $\left(\mathcal{S}^{ \pm}\right)^{*}$ by concatenating the labels of the edges along the path.

We require that the labelling is reduced, in the sense that no vertex has two incident outgoing edges with the same label, and no vertex has two incident incoming edges with the same label. This implies that the word read on an immersed path is freely reduced and, hence, an element of $\langle\mathcal{S}\rangle$. Also, the word read on an immersed cycle is cyclically reduced.

Let $\mathcal{R}$ be the set of words in $\langle\mathcal{S}\rangle$ read on embedded cycles in $\Gamma$. Note that this definition implies that elements of $\mathcal{R}$ are cyclically reduced and that $\mathcal{R}$ is closed under inversion and cyclic permutation of its elements.

Definition 2.1 (Group defined by a labelled graph). The group $G$ defined by a reduced $\mathcal{S}$-labelled graph $\Gamma$ is the group $G(\Gamma):=\langle\mathcal{S} \mid \mathcal{R}\rangle$.

The notion of a group defined by a labelled graph first appeared in Rips and Segev's construction of torsion-free groups without the unique-product property [43].

Definition 2.2 (Piece). A piece is a labelled path graph $p$ that admits two distinct label-preserving maps $\phi_{1}, \phi_{2}: p \rightarrow \Gamma$ such that there is no label-preserving automorphism $\psi$ of $\Gamma$ with $\phi_{2}=\psi \circ \phi_{1}$.

Definition 2.3 $\left(G r^{\prime}(\lambda)\right.$ and $C^{\prime}(\lambda)$ conditions). Let $\Gamma$ be a reduced labelled graph, and let $\lambda>0$.

$\Gamma$ is $G r^{\prime}(\lambda)$-labelled if whenever $p$ is a piece contained in a simple cycle $c$ of $\Gamma$ then $|p|<\lambda|c|$.

$\Gamma$ is $C^{\prime}(\lambda)$-labelled if it is $G r^{\prime}(\lambda)$-labelled and, in addition, every label-preserving automorphism of $\Gamma$ restricts to the identity on every connected component with non-trivial fundamental group.

A presentation $\langle\mathcal{S} \mid \mathcal{R}\rangle$ satisfies the classical $C^{\prime}(\lambda)$-condition if the disjoint union of cycle graphs labelled by the elements of $\mathcal{R}$ is a $G r^{\prime}(\lambda)$-labelled graph.

Actually, every group is defined by a $G r^{\prime}(1 / 6)$-labelled graph: simply take $\Gamma$ to be its Cayley graph with respect to any generating set of the group [27, Example 2.2]. Therefore, general statements about groups defined by $G r^{\prime}(1 / 6)$-labelled graphs either require that some additional condition be imposed on $\Gamma$ or are tautologically true when $\Gamma=\operatorname{Cay}(G(\Gamma), \mathcal{S})$. 
A subspace $Y$ of a geodesic metric space is convex if every geodesic segment between points of $Y$ is contained in $Y$.

Lemma 2.4 ([31, Lemma 2.15]). Let $\Gamma$ be a $G r^{\prime}(1 / 6)$-labelled graph. Let $\Gamma_{i}$ be a component of $\Gamma$. For any choice of a vertex $x \in X:=\operatorname{Cay}(G(\Gamma), \mathcal{S})$ and any vertex $y \in \Gamma_{i}$ there is a unique label-preserving map $\Gamma_{i} \rightarrow X$ that takes $y$ to $x$, and this map is an isometric embedding with convex image.

Definition 2.5 (Embedded component). An embedded component $\Gamma_{0}$ of $\Gamma$ refers to the image of an isometric embedding of some $\Gamma_{i}$ into $X:=\operatorname{Cay}(G(\Gamma), \mathcal{S})$ via a label-preserving map. Equivalently, it is a $G(\Gamma)$-translate in $X$ of the image of $\Gamma_{i}$ under the unique label-preserving map determined by an arbitrary choice of basepoints in $X$ and $\Gamma_{i}$.

We consider a graph $\Gamma$ as a sequence $\left(\Gamma_{i}\right)_{i}$ of its connected components.

2.2. Contraction terminology. Recall Definition 1.1. In [7] we considered, more generally, almost closest point projections $x \mapsto\{y \in Y \mid d(x, y) \leqslant d(x, Y)+\epsilon\}$ to ensure the empty set is not in the image of the projection. That is unnecessary in this paper as we are in the case that $Y$ is a subgraph of a graph $X$, which guarantees $\emptyset \notin \operatorname{Im} \pi$. Here, and from now on, $\pi: X \rightarrow 2^{Y}$ denotes closest point projection to $Y$.

We say a geodesic $\alpha$ in $\operatorname{Cay}(G(\Gamma), \mathcal{S})$ is locally $\left(\rho_{1}, \rho_{2}\right)$-contracting if, for each embedded component $\Gamma_{0}$ of $\Gamma$ such that $\Gamma_{0} \cap \alpha$ is non-empty, closest point projection in $\Gamma_{0}$ of $\Gamma_{0}$ to $\Gamma_{0} \cap \alpha$ is $\left(\rho_{1}, \rho_{2}\right)$-contracting.

We say a geodesic is uniformly locally contracting if there exist $\rho_{1}$ and $\rho_{2}$ such that it is locally $\left(\rho_{1}, \rho_{2}\right)$-contracting. We add 'uniform' here to stress that the intersection of the geodesic with each embedded component of $\Gamma$ is contracting with respect to the same contraction functions. Similarly, a geodesic is uniformly locally sublinearly contracting if it is locally $\left(r, \rho_{2}\right)$-contracting, and is uniformly locally strongly contracting is it locally $\left(r, \rho_{2}\right)$-contracting for $\rho_{2}$ bounded.

\section{Classification of quadrangles}

In this section, we establish geometric results that will let us prove our theorems about contraction in graphical small cancellation groups. In particular, we provide a complete classification of the geodesic quadrangles in the Cayley graph of a group defined by a $G r^{\prime}(1 / 6)$-labelled graph. The main technical result to be used in our subsequent investigation will be recorded in Proposition 3.20.

3.1. Combinatorial geodesic polygons. One of the main tools of small cancellation theory are so-called 'van Kampen diagrams'.

Definition 3.1 (Diagram). A (disc) diagram is a finite, simply-connected, 2-dimensional CW complex with an embedding into the plane, considered up to orientation-preserving homeomorphisms of the plane. It is $\mathcal{S}$-labelled if its directed edges are labelled by elements in $\mathcal{S}$. It is a diagram over $\mathcal{R}$ if it is $\mathcal{S}$-labelled and the word read on the boundary of each $2-$-cell belongs to $\mathcal{R}$. A diagram is simple if it is homeomorphic to a disc.

If $D$ is a diagram over $\mathcal{R}, b$ is a basepoint in $D$, and $g$ is an element of $G=\langle\mathcal{S} \mid \mathcal{R}\rangle$, then there exists a unique label-preserving map from the 1 -skeleton of $D$ into $\operatorname{Cay}(G, \mathcal{S})$ taking $b$ to $g$. In general, this map need not be an immersion.

An arc in a diagram $D$ is a maximal path of length at least 1 all of whose interior vertices have valence 2 in $D$. An interior arc is an arc whose interior is contained in the interior of $D$. An exterior arc is an arc contained in the boundary of $D$. A face is the image of a closed 2-cell of $D$. If $\Pi$ is a face, its interior degree $i(\Pi)$ is the number of 
interior arcs in its boundary. Likewise, its exterior degree $e(\Pi)$ is the number of exterior arcs. An interior face is one with exterior degree 0; an exterior face is one with positive exterior degree.

If $D$ is a finite, simply connected, planar, 2-dimensional CW-complex whose boundary is written as a concatenation of immersed subpaths $\gamma_{1}, \ldots, \gamma_{k}$, which we call sides of $D$, then there is a unique, up to orientation-preserving homeomorphism of $\mathbb{R}^{2}$, embedding $\phi: D \rightarrow \mathbb{R}^{2}$ such that the concatenation of the $\phi\left(\gamma_{i}\right)$ is the positively oriented boundary $\partial \phi(D)$. This claim follows easily from the Schoenflies Theorem. Thus, $\left(D,\left(\gamma_{i}\right)_{i}\right)$ uniquely determines a (not necessarily simple) diagram $\phi(D)$. We call $\phi$ the canonical embedding of $\left(D,\left(\gamma_{i}\right)_{i}\right)$. Having said this once, we omit $\phi$ from the notation and conflate $D$ and the $\gamma_{i}$ with their $\phi$-images.

Definition $3.2((3,7)$-diagram). A $(3,7)$-diagram is a diagram such that every interior vertex has valence at least three and every interior face has interior degree at least seven.

Definition 3.3 (Combinatorial geodesic polygon [31, Definition 2.11]). A combinatorial geodesic $n$-gon $\left(D,\left(\gamma_{i}\right)_{i}\right)$ is a $(3,7)$-diagram $D$ whose boundary is a concatenation of immersed subpaths $\gamma_{0}, \ldots, \gamma_{n-1}$ such that each boundary face whose exterior part is a single arc that is contained in one of the sides $\gamma_{i}$ has interior degree at least 4 . A valence 2 vertex that belongs to more than one side is called a distinguished vertex. A face whose exterior part contains an arc not contained in one of the sides is a distinguished face.

The ordering of the sides of a combinatorial geodesic $n$-gon is considered up to cyclic permutation, with subscripts modulo $n$. We also refer to 'the combinatorial geodesic $n$-gon $D$ ' when the sides are clear from context or irrelevant. We can also say 'combinatorial geodesic polygon' when the number of sides is irrelevant. Following common usage, 2-gons, 3-gons, and 4-gons will respectively be denominated bigons, triangles, and quadrangles.

If $D$ is a simple combinatorial geodesic $n$-gon then every distinguished face contains a distinguished vertex, so there are at most $n$ distinguished faces.

We record the following crucial fact about diagrams over graphical small cancellation presentations. In the following, $\mathcal{R}$ is the set of labels of simple cycles on a $G r^{\prime}(1 / 6)$-graph $\Gamma$ labelled by the set $\mathcal{S}$, and $X:=\operatorname{Cay}(G(\Gamma), \mathcal{S})$.

Lemma 3.4 ([27, Lemma 2.13]). Let $\Gamma$ be a $G r^{\prime}(1 / 6)$-labelled graph, and let $w \in\langle\mathcal{S}\rangle$ represent the identity in $G(\Gamma)$. Then, there exists an $\mathcal{S}$-labelled diagram over $\mathcal{R}$ with boundary word $w$ in which every interior arc is a piece.

The sides of a combinatorial geodesic polygon are not assumed to be geodesic. The definition and choice of terminology are motivated by the following proposition. An $n$-gon $P$ in $X$ is a closed edge path that decomposes into immersed simplicial subpaths $\gamma_{0}^{\prime}, \ldots, \gamma_{n-1}^{\prime}$, which are called sides of $P$.

Proposition 3.5. If $P$ is an $n-$ gon in $X$ with sides $\gamma_{0}^{\prime}, \ldots, \gamma_{n-1}^{\prime}$ that are geodesics then there is an $\mathcal{S}$-labelled diagram $D$ over $\mathcal{R}$ with sides $\gamma_{0}, \ldots, \gamma_{n-1}$ such that for each $0 \leqslant i<n$ the word of $\langle\mathcal{S}\rangle$ read on $\gamma_{i}$ is the same as the word read on $\gamma_{i}^{\prime}$. Furthermore, we can choose $D$ in such a way that after forgetting interior vertices of valence 2 , we obtain a combinatorial geodesic $n-$ gon $\left(D,\left(\gamma_{i}\right)_{i}\right)$

Here, forgetting one interior vertex of valence 2 means replacing its two incident edges by a single one. Note that, when performing this operation, we consider $D$ merely as (unlabelled) diagram, i.e. we ignore the orientations and labels of edges. Forgetting interior vertices of valence 2 means iterating this operation, such that we end up with a diagram without interior vertices of valence 2 . 
Proof. The existence of an $\mathcal{S}$-labelled diagram over $\mathcal{R}$ whose boundary label matches the label of $P$ is the well-known van Kampen Lemma. Lemma 3.4 guarantees that the diagram can be chosen such that all interior arcs are pieces. The small cancellation condition then implies interior faces have interior degree at least 7 . If there is a face $\Pi$ with $e(\Pi)=1$ whose exterior part is contained in a single $\gamma_{i}$, then the exterior part is a geodesic. Thus, the length of the interior part is at least half of the length of $\partial \Pi$. Since interior arcs are pieces, the small cancellation condition implies there must be at least four of them to account for half the length of $\partial \Pi$. Now if we forget interior vertices of valence 2 , we have the desired combinatorial geodesic polygon.

Remark 3.6. The word $w \in\langle\mathcal{S}\rangle$ read on a cycle in $X$ represents the trivial element in $G(\Gamma)$. The combinatorial geodesic $n$-gon of Proposition 3.5 is a special type of van Kampen diagram witnessing the triviality of the word $w$ labelling an $n$-gon in $X$ whose sides are geodesics. In the remainder of Section 3 we make combinatorial arguments about arbitrary $(3,7)$-diagrams, not necessarily $\mathcal{S}$-labelled diagrams over $\mathcal{R}$. In Section 4 we use Proposition 3.5 to apply results of this section to graphical small cancellation groups.

We record an equivalent formulation of the Euler characteristic formula for certain diagrams. Recall that for a face $\Pi$ of a diagram, $e(\Pi)$ is the exterior degree of $\Pi$, which is the number of exterior arcs in its boundary. Similarly, $i(\Pi)$ is the interior degree of $\Pi$.

Lemma 3.7 (Strebel's curvature formula, [48, p.253]). Let D be a simple diagram without vertices of degree 2. Then:

$$
\begin{aligned}
6 & =2 \sum_{v}(3-d(v)) \\
& +\sum_{e(\Pi)=0}(6-i(\Pi))+\sum_{e(\Pi)=1}(4-i(\Pi))+\sum_{e(\Pi) \geqslant 2}(6-2 e(\Pi)-i(\Pi)) .
\end{aligned}
$$

Here $d(v)$ denotes the degree of a vertex $v$.

It readily follows from Lemma 3.7 that any $(3,7)$-diagram with more than one face has at least 2 faces with exterior degree 1 and interior degree at most 3. (This is usually known as Greendlinger's lemma.) Therefore:

Lemma 3.8. The sides of a combinatorial geodesic polygon are embedded, and every combinatorial geodesic polygon has at least two sides.

The same argument gives the following well-known fact, which greatly simplifies many considerations:

Lemma 3.9. Let $D$ be a $(3,7)$-diagram. Then any face is simply connected.

We also state an immediate consequence of [28, Lemma 4.14]:

Lemma 3.10. If $\Pi$ is a face of a combinatorial geodesic polygon $D$ and $\alpha$ is a side of $D$ then $\Pi \cap \alpha$ is empty or connected. If $\Pi_{1}, \ldots, \Pi_{k}$ is a sequence of faces of a combinatorial geodesic polygon $D$ such that $\Pi_{i} \cap \Pi_{i+1} \neq \emptyset$ for all $1 \leqslant i<k$ and $\alpha$ is a side of $D$ such that $\Pi_{i} \cap \alpha \neq \emptyset$ for all $i$ then $\cup_{1 \leqslant i \leqslant k} \Pi_{i} \cap \alpha$ is connected.

Definition 3.11 (Degenerate). A combinatorial geodesic $n$-gon $\left(D,\left(\gamma_{i}\right)_{i}\right)$ is degenerate if there exists an $i$ such that $D, \gamma_{0}, \ldots, \gamma_{i} \gamma_{i+1}, \ldots, \gamma_{n-1}$ is a combinatorial geodesic $(n-1)$-gon. In this case the terminal vertex of $\gamma_{i}$ is called a degenerate vertex.

It will be useful to minimize the number of sides of a diagram $D$ by replacing a degenerate combinatorial geodesic $n$-gon $\left(D,\left(\gamma_{i}\right)_{i}\right)$ with a non-degenerate combinatorial geodesic $k$-gon $\left(D,\left(\gamma_{i}^{\prime}\right)_{i}\right)$ for some $k<n$. 
3.2. Reducibility. In this section we define operations for combining and reducing combinatorial geodesic $n$-gons. We stress that the setting is only combinatorial - these operations need not preserve the property of being a diagram over $\mathcal{R}$.

First note that if $\left(D,\left(\gamma_{i}\right)_{i}\right)$ is a combinatorial geodesic $n$-gon, and if $D^{\prime}$ is obtained from $D$ by subdividing an edge, then $\left(D^{\prime},\left(\gamma_{i}\right)_{i}\right)$ is still a combinatorial geodesic $n$-gon. The new vertex produced by subdivision is a non-distinguished vertex of valence 2 . Conversely, if $v$ is a non-distinguished vertex of valence 2 then we can 'forget' it by replacing the two incident edges with a single edge.

If $D$ is simple and non-degenerate then by forgetting all non-distinguished vertices of valence 2 we can arrange that the distinguished vertices are exactly the vertices of valence 2 and all other vertices have valence at least 3 .

Definition 3.12 (Reducible). A combinatorial geodesic $l$-gon $P$ is reducible if it admits a vertex, edge, or face reduction, as defined below, see Figure 4. It is irreducible otherwise.
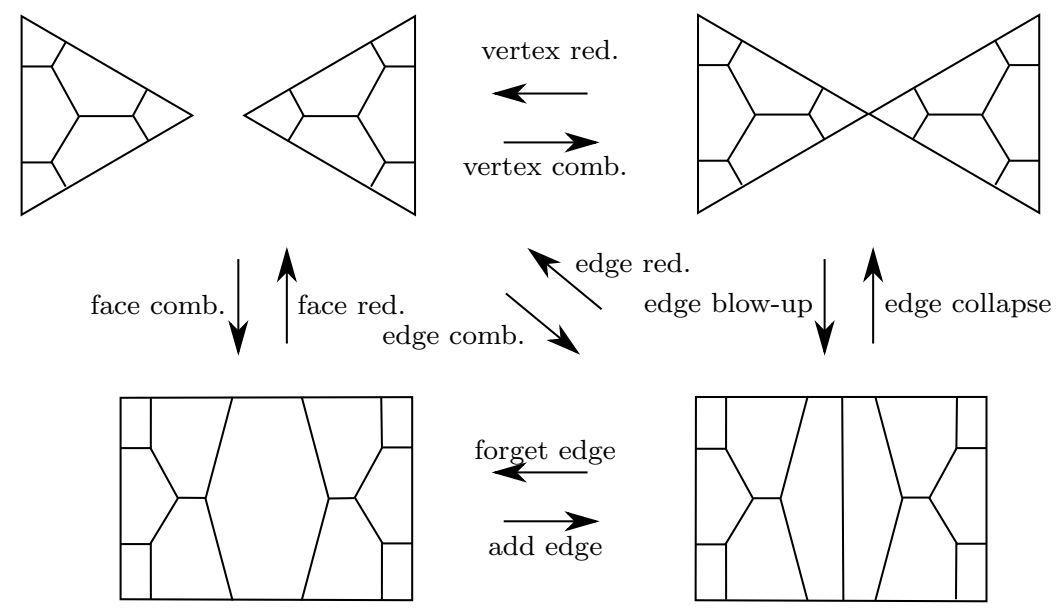

Figure 4. Combination and reduction

In all of the following cases, let $\left(D,\left(\gamma_{i}\right)_{i}\right)$ be a combinatorial geodesic $n$-gon, and let $\left(D^{\prime},\left(\gamma_{i}^{\prime}\right)_{i}\right)$ be a combinatorial geodesic $n^{\prime}$-gon.

3.2.1. Vertex reduction. Suppose $v \in D$ is a separating vertex that is in the boundary of exactly two faces and that these two faces are the only maximal cells containing $v$. Suppose that there are exactly two sides, $\gamma_{i}$ and $\gamma_{j}$, containing $v$, which is necessarily true if $\left(D,\left(\gamma_{i}\right)_{i}\right)$ is non-degenerate. Let $\gamma_{i}^{-v}$ denote the initial path of $\gamma_{i}$ ending at $v$, and let $\gamma_{i}^{v+}$ denote the terminal path of $\gamma_{i}$ beginning at $v$, and similarly for $\gamma_{j}$. Define the vertex reduction of $\left(D,\left(\gamma_{i}\right)_{i}\right)$ at $v$ to be the two combinatorial geodesic polygons whose underlying CWcomplexes are each $D$ minus one of the complementary components of $D \backslash v$, respectively, and whose sides are, respectively, $\gamma_{i}^{v+}, \gamma_{i+1}, \ldots, \gamma_{j-1}, \gamma_{j}^{-v}$ and $\gamma_{j}^{v+}, \gamma_{j+1}, \ldots \gamma_{i-1}, \gamma_{i}^{-v}$.

Note that each of the resulting combinatorial polygons contains a distinguished vertex corresponding to $v$.

The inverse operation of vertex reduction we denominate a vertex combination. Suppose that $v \in D$ is a distinguished vertex that is contained in the boundary of a single face and is not contained in any other maximal cell. Then there is some $i$ such that $v=\gamma_{i} \cap \gamma_{i+1}$. Make corresponding assumptions for $v^{\prime}=\gamma_{i^{\prime}}^{\prime} \cap \gamma_{i^{\prime}+1}^{\prime} \subset D^{\prime}$. The vertex combination of $\left(D,\left(\gamma_{i}\right)_{i}\right)$ and $\left(D^{\prime},\left(\gamma_{i}^{\prime}\right)_{i}\right)$ at $v$ and $v^{\prime}$ is the combinatorial geodesic $\left(n+n^{\prime}-2\right)$-gon whose underlying CW-complex is the wedge sum of $D$ and $D^{\prime}$ at $v$ and $v^{\prime}$, and whose sides are:

$$
\gamma_{0}, \ldots, \gamma_{i-1}, \gamma_{i} \gamma_{i^{\prime}+1}^{\prime}, \gamma_{i^{\prime}+2}^{\prime}, \ldots, \gamma_{i^{\prime}-1}^{\prime}, \gamma_{i^{\prime}}^{\prime} \gamma_{i+1}, \gamma_{i+1}, \ldots, \gamma_{n-1}
$$


This is the unique way to define the sides so that the canonical embeddings of $\left(D,\left(\gamma_{i}\right)_{i}\right)$ and $\left(D^{\prime},\left(\gamma_{i}^{\prime}\right)_{i}\right)$ factor through inclusion into the wedge sum and the canonical embedding of the resulting combinatorial geodesic $\left(n+n^{\prime}-2\right)$-gon.

3.2.2. Edge reduction. Suppose $e \subset D$ is an interior edge of $D$ such that the boundary of $e$ is contained in the boundary of $D$. Suppose further that the only maximal cells that intersect $e$ are the two faces that intersect its interior. The hypotheses imply that $e$ separates $D$ into two components, and the $(3,7)$-condition implies that $e$ has two distinct boundary vertices. Suppose that each of these boundary vertices belongs to exactly one side, which is necessarily true if $\left(D,\left(\gamma_{i}\right)_{i}\right)$ is non-degenerate. Define the edge reduction of $\left(D,\left(\gamma_{i}\right)_{i}\right)$ at $e$ to be the two combinatorial geodesic polygons obtained by collapsing $e$ to a vertex and then performing vertex reduction at the resulting vertex.

The inverse operation to edge reduction we denominate edge combination. Suppose each of $v \in D$ and $v^{\prime} \in D^{\prime}$ is a distinguished vertex that is contained in a single face and in no other maximal cell. First perform a vertex combination at $v$ and $v^{\prime}$ and then blow up the wedge point to an interior edge, while keeping the same sides. As before, we require that $v$ and $v^{\prime}$ each belong to a single face and no other maximal cell, which implies that the resulting combinatorial geodesic polygon is uniquely determined.

3.2.3. Face reduction. Suppose $\Pi \subset D$ is a face with $e(\Pi) \geqslant 2$. Suppose that there are boundary edges $e$ and $e^{\prime}$ of $\Pi$ that are boundary edges of $D$ such that removing the union of the interiors of $\Pi, e$, and $e^{\prime}$ separates $D$ into two components, $D_{1}$ and $D_{2}$. Suppose that $e$ and $e^{\prime}$ each intersect only one side of $D$, which is necessarily true if $\left(D,\left(\gamma_{i}\right)_{i}\right)$ is non-degenerate. Finally, suppose that $D_{1}$ and $D_{2}$ each contain a distinguished vertex. Define the face reduction of $\left(D,\left(\gamma_{i}\right)_{i}\right)$ at $\left(\Pi, e, e^{\prime}\right)$, or at $\Pi$, when $e$ and $e^{\prime}$ are clear, to be the two combinatorial geodesic polygons obtained by subdividing $e$ and $e^{\prime}$, subdividing $\Pi$ by adding a new edge connecting the subdivision points of $e$ and $e^{\prime}$, and then performing an edge reduction on this new edge.

The inverse operation to face reduction, which we denominate face combination, is to first perform edge combination, which results in a new interior edge in the boundary of exactly two faces, and then forget this new edge, replacing the two incident faces by a single face, and replacing the four resulting edges by two.

3.3. Combinatorial geodesic bigons and triangles. We state Strebel's classification of combinatorial geodesic bigons and triangles. Let us stress again that we are working in the combinatorial setting, cf Remark 3.6. Strebel's original statement includes that the diagram $D$ comes from a small cancellation presentation, but what is actually used in the proof are the properties of the diagram that we have encapsulated in the definition of 'combinatorial geodesic polygon', Definition 3.3. This observation was first made in [31, Section 2.5 and Remark 4.7] and [28, Lemma 4.7].

Theorem 3.13 (Strebel's classification ${ }^{1}$, [48, Theorem 43]). Let D be a simple diagram that is not a single face.

- If $D$ is a combinatorial geodesic bigon, then $D$ has shape $\mathrm{I}_{1}$ in Figure 5.

- If $D$ is a combinatorial geodesic triangle, then $D$ has one of the shapes $\mathrm{I}_{2}, \mathrm{I}_{3}$, II, $\mathrm{III}_{1}$, IV, or V in Figure 5.

Note that shapes $\mathrm{I}_{2}$ and $\mathrm{I}_{3}$ degenerate to combinatorial geodesic bigons.

\footnotetext{
${ }^{1}$ Strebel also considers a second definition of combinatorial geodesic polygon that yields one additional shape $\mathrm{III}_{2}$. This is not relevant for us, but we retain the subscript for shape $\mathrm{III}_{1}$ for consistency with Strebel's notation.
} 


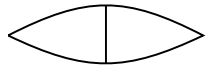

$\mathrm{I}_{1}$

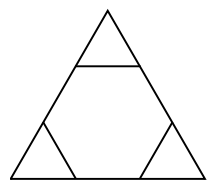

II

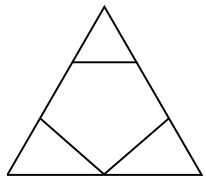

$\mathrm{III}_{1}$

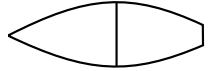

$\mathrm{I}_{2}$

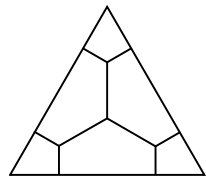

IV

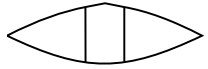

$\mathrm{I}_{3}$

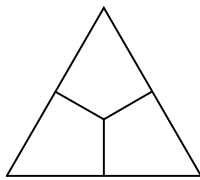

$\mathrm{V}$

FigURE 5. Strebel's classification of combinatorial geodesic bigons and triangles.

Each of these shapes represents an infinite family of combinatorial geodesic bigons or triangles obtained by performing face combination at a non-degenerate, distinguished vertex with a shape $\mathrm{I}_{1}$ bigon arbitrarily many times. Figure 6 shows alternate examples of each shape.

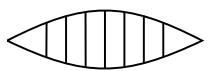

$\mathrm{I}_{1}$

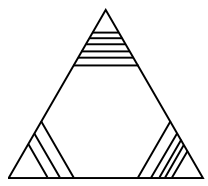

II

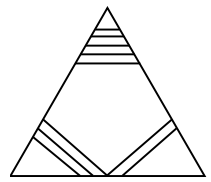

$\mathrm{III}_{1}$

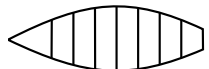

$\mathrm{I}_{2}$

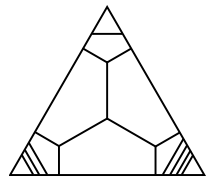

IV

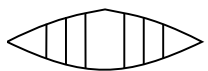

$\mathrm{I}_{3}$

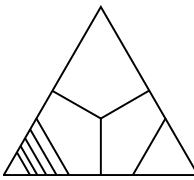

$\mathrm{V}$

FiguRE 6. Alternate examples of each shape.

3.4. Special combinatorial geodesic quadrangles. In this section diagram faces are labelled with their contribution to the curvature sum (Lemma 3.7) if this contribution is non-zero.

Definition 3.14 (Special). A combinatorial geodesic $n$-gon, for $n>2$, is special if it is simple, non-degenerate, irreducible, and every non-distinguished vertex has valence 3 .

The only special combinatorial geodesic triangles are the representatives of shapes IV and $\mathrm{V}$ pictured in Figure 5. In this section we classify special combinatorial geodesic quadrangles.

Let $D$ be a special combinatorial geodesic polygon. Simplicity and trivalence imply that $\Pi \cap \partial D$ is a disjoint union of arcs, for each face $\Pi$. Irreducibility implies $\Pi \cap \partial D$ consists of at most one arc. Non-degeneracy implies that every distinguished face has exactly one distinguished vertex and either 2 or 3 interior arcs.

The curvature formula of Lemma 3.7 can be simplified as follows.

Lemma 3.15 (Special curvature formula). Let $D$ be a special combinatorial geodesic polygon that is not a single face. Then:

$$
6=\sum_{e(\Pi)=0}(6-i(\Pi))+\sum_{e(\Pi)=1}(4-i(\Pi))
$$

Proof. By definition, every non-distinguished vertex has valence 3. To apply Lemma 3.7, we must address the possible existence of degree 2 vertices in the boundary: we may 
iteratively remove such vertices, always replacing the two adjacent edges by a single edge. Since $D$ is not a single face, this makes sense for every degree 2 vertex, and we thus remove all degree 2 vertices. Since the degree of a face counts arcs, not edges, the operation does not alter the sum. The formula follows by applying Lemma 3.7, and noting, as a consequence of irreducibility, that every face has exterior degree at most 1.

For the remainder of this section, let $D$ be a special combinatorial geodesic quadrangle. Then $D$ has exactly four distinguished faces, each of which contributes either 1 or 2 to the curvature sum, and every other face makes a non-positive contribution. Let $D_{k}$ refer to the set of distinguished faces of $D$ that contribute $k$ to the curvature sum, ie, with $e(\Pi)=1$ and $i(\Pi)=4-k$.

An ordinary face will refer to a non-distinguished face $\Pi$ with $e(\Pi)=1$ and $i(\Pi)=4$, which contributes 0 to the curvature sum. An extraordinary face will refer to a nondistinguished face $\Pi$ with $e(\Pi)=0$ or with $e(\Pi)=1$ and $i(\Pi)>4$. Note that if $i(\Pi)>6$, we must have $e(\Pi)=0$.

3.4.1. Zippers. Ordinary faces can fit together to make arbitrarily long sequences of subsequent faces we call zippers, as in Figure 7.

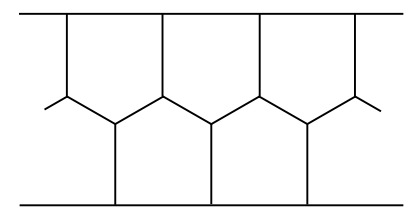

Figure 7. A zipper.

The ordinary faces in a zipper are called teeth. We define a zipper $Z$ with zero teeth to be three consecutive interior edges that separate the diagram into two parts, each of which contains two distinguished faces. For example, the bold edges of Figure 8 form a zipper with zero teeth. Since $D$ is special, the two interior edges incident to the interior vertices of $Z$ and not belonging to $Z$ must be contained in opposite complementary components of $Z$, otherwise $D$ would admit a face reduction.

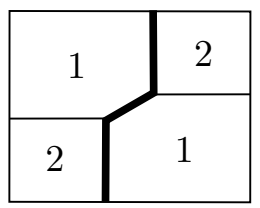

FigURE 8. Zipper with zero teeth (in bold).

Using a symmetry argument, we show that the portions of the diagram on opposite sides of a zipper each contribute 3 to the curvature sum: first, consider a zipper $Z$ of length 0 . Then the two interior edges incident at $Z$ are not on the same side of $Z$, for otherwise we would have a face with exterior degree at least 2 (and hence face-reducibility) or only one distinguished face on that side. Now assume that one of the two sides $S$ contributes $k \neq 3$ to the curvature sum. Then we may rotate a copy of $S$ by 180 degrees and attach it to $S$ by identifying the respective copies of $Z$, thus obtaining a special combinatorial quadrangle for which the curvature formula amounts to $2 k \neq 6$; a contradiction. The case of an arbitrary zipper $Z$ now follows similarly by attaching a rotated (or in the case that $Z$ has an odd number of faces reflected) copy of $S$ to $S \cup Z$. 
We need to see how to terminate a zipper. Let $\Pi$ be the face with two edges on the zipper. If $\Pi$ is ordinary then the zipper just gets longer, so assume not. One possibility is that $\Pi$ is distinguished, in which case it is a $D_{1}$ and there is only one other face, which is a $D_{2}$. Otherwise, since the end of the zipper containing $\Pi$ must contribute 3 to the curvature sum, we must have $i(\Pi)=5$ and $e(\Pi)=1$, both distinguished faces must be $D_{2}$ 's, and the other two faces sharing edges with $\Pi$ must be ordinary.

The two possibilities are shown in Figure 9. In conclusion:

Lemma 3.16. There are six infinite families of configurations of special combinatorial geodesic quadrangles containing zippers, determined by the choice of two zipper ends from Figure 9 and the parity of the number of teeth.

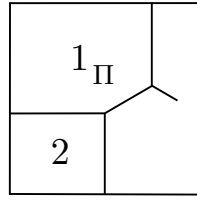

(1) Zipper end 1.

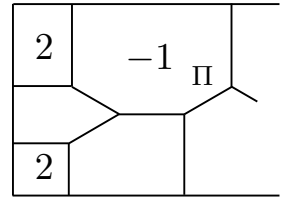

(2) Zipper end 2.

Figure 9. Terminating a zipper

\subsubsection{Extraordinary configurations.}

Lemma 3.17. The six configurations shown in Figure 10 are the only special combinatorial geodesic quadrangles containing an extraordinary face and no zipper.

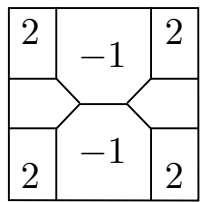

$\mathrm{E}_{5}$

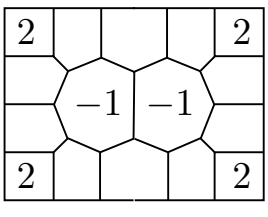

$\mathrm{E}_{7 b}$
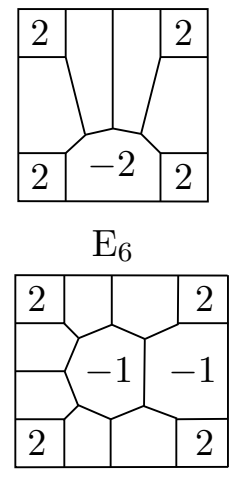

$\mathrm{E}_{7 c}$
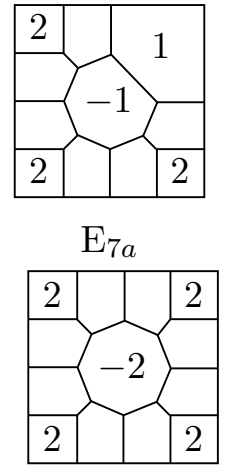

$\mathrm{E}_{8}$

FiguRE 10. Extraordinary special combinatorial geodesic quadrangles.

Proof. Let $D$ be a special combinatorial geodesic quadrangle without zippers. First, suppose that $D$ contains no interior faces.

Case $\mathrm{E}_{5}: D$ contains an extraordinary face $\Pi$ with $i(\Pi)=5$. In this case $\Pi$ contributes -1 to the curvature sum. Consider the third interior edge $e$ of $\Pi$. Let $\Pi^{\prime}$ be the face on the opposite side of $e$. If $e$ is the second interior edge of $\Pi^{\prime}$ then we get a zipper, so it must be at least the third. (It cannot be the first, for this would give a vertex of degree at least 4.)

By symmetry, we see that $i\left(\Pi^{\prime}\right) \geqslant 5$. As $\Pi^{\prime}$ contributes at least -1 to the curvature sum and is not interior, we deduce $i\left(\Pi^{\prime}\right)=5$ and $e\left(\Pi^{\prime}\right)=1$, with $e$ the third interior edge of $\Pi^{\prime}$. This then implies that the distinguished faces are all $D_{2}$ 's, and every other face is ordinary. There are two ordinary faces bordering both $\Pi$ and $\Pi^{\prime}$. Since no face has more than one exterior edge, we must then fill in the four $D_{2}$ 's on the corners. 
Case $\mathrm{E}_{6}: D$ contains an extraordinary face $\Pi$ with $i(\Pi)=6$. In this case $\Pi$ contributes -2 to the curvature sum, so the distinguished faces are all $D_{2}$ 's, and every other face is ordinary. Consider the third interior vertex of $\Pi$. Let $e$ be the edge incident to this vertex that does not belong to $\Pi$. If $e$ does not have a vertex on the boundary then there is a zipper contained in the boundary of $\Pi \cup \Pi^{\prime}$, where $\Pi^{\prime}$ is either one of the ordinary faces with side $e$. Since we have assumed no zippers, $e$ does have a boundary vertex, and there is a unique way to fill in the rest of $D$ with ordinary faces and $D_{2}$ 's.

Now we move on to the interior face cases. According to the curvature formula of Lemma 3.7, interior faces have either 7 or 8 sides.

Case $\mathrm{E}_{8}: D$ contains an extraordinary face $\Pi$ with $i(\Pi)=8$. In this case, $e(\Pi)=0$, and $\Pi$ contributes -2 to the curvature sum, so all four distinguished faces are $D_{2}$ and every other face is ordinary. Since the interior sides of a $D_{2}$ have a vertex on the boundary, they cannot share an edge with $\Pi$, so every face sharing an edge with $\Pi$ is ordinary. There is only one way to pack 8 ordinary faces around $\Pi$, up to symmetry, and this determines the placement of the four $D_{2}$ 's.

Case $\mathrm{E}_{7}: D$ contains an extraordinary face $\Pi$ with $i(\Pi)=7$. In this case, $e(\Pi)=0$, and $\Pi$ contributes -1 to the curvature sum. The distinguished faces are therefore either three $D_{2}$ 's and one $D_{1}$ or four $D_{2}$ 's. If there are three $D_{2}$ 's and one $D_{1}$ then every other face is ordinary. A $D_{2}$ cannot share an edge with an interior face, so $\Pi$ has at least 6 edges that are shared by ordinary faces.

If there are four $D_{2}$ 's then none of them share a face with $\Pi$, and there is exactly one other face that is not ordinary.

In either case, $\Pi$ has at least 6 edges that are shared by ordinary faces. Let $\Pi_{1}, \ldots, \Pi_{7}$ be the consecutive faces sharing an edge with $\Pi$, and assume all except possibly $\Pi_{7}$ are ordinary. Let $e$ be the edge shared by $\Pi_{1}$ and $\Pi_{7}$. If $e$ has a vertex on the boundary then there is only one way to fit 6 ordinary faces around $\Pi$. In this case, $\Pi_{7}$ is a $D_{1}$, and the configuration is shown in Figure 11(1).

If $e$ does not have a vertex on the boundary then, again, there is only one way to fit six ordinary faces around $\Pi$, shown in Figure $11(2)$. We see that $i\left(\Pi_{7}\right) \geqslant 5$. Since $\Pi_{7}$ contributes at least -1 to the curvature sum, the two possibilities are $i\left(\Pi_{7}\right)=7$ and $e\left(\Pi_{7}\right)=0$ or $i\left(\Pi_{7}\right)=5$ and $e\left(\Pi_{7}\right)=1$. In both cases, the remaining distinguished faces are $D_{2}$ 's, all other faces are ordinary, and there is a unique way to complete the 4-gon. These are types $\mathrm{E}_{7 b}$ and $\mathrm{E}_{7 c}$, respectively, of Figure 10.

\begin{tabular}{|l|l|l|l|}
\hline 2 & $\Pi_{1}$ & $e$ & 1 \\
\hline$\Pi_{2}$ & & $\Pi_{7}$ \\
\hline$\Pi_{3}$ & -1 & $\Pi_{6}$ \\
\hline 2 & $\Pi_{4}$ & $\Pi_{5}$ & 2 \\
\hline
\end{tabular}

(1) Case $e$ does have boundary vertex.

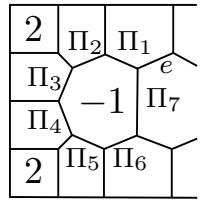

(2) Case $e$ does not have boundary vertex.

FiguRE 11. Interior 7-gon.

\subsubsection{Classification of special combinatorial geodesic quadrangles.}

Theorem 3.18. Every special combinatorial geodesic quadrangle is either one of the six extraordinary configurations of Lemma 3.17 or belongs to one of the six zippered families of Lemma 3.16.

The theorem is proven by Lemma 3.17, Lemma 3.16, and the following: 
Lemma 3.19. Every special combinatorial geodesic quadrangle contains an extraordinary face or a zipper.

Proof. Suppose $D$ is a special combinatorial geodesic quadrangle that does not contain an extraordinary face. Then no face makes a negative contribution to the curvature sum, so $D$ is composed of two $D_{2}$ 's, two $D_{1}$ 's, and some number of ordinary faces.

Pick a side of the quadrangle. Let $S$ be the union of faces along the side. Let $A$ be the union of interior edges separating $S$ from $D \backslash S$. Since every vertex has valence 3 , both $S$ and $D \backslash S$ are connected, and each contains two distinguished faces.

Consider the edges incident to interior vertices of $A$. Each one is contained either in $S$ or in $D \backslash S$. At least one is contained in $S$ and one in $D \backslash S$, since each side contains two distinguished vertices and no face separates $D$. Two consecutive edges cannot point into $D \backslash S$, because the face $\Pi \subset S$ containing the edge between them would either be extraordinary, contradicting the hypothesis, or a distinguished face with interior degree at least four, contradicting non-degeneracy.

Two consecutive edges cannot point into $S$, because the face $\Pi \subset S$ between them would be non-distinguished with $i(\Pi)=3$. Therefore the edges along $A$ alternate, and $A$ has length at least 3 .

If $A$ consists of 3 edges then it is a zipper, and we are done, so suppose it consists of at least 4 edges.

If the first face $\Pi$ adjacent to $A$ on the $D \backslash S$ side is distinguished then we get a zipper, so suppose it is not. Let $\Pi^{\prime}$ be the next face along $A$ on the $D \backslash S$ side. The two possibilities are shown in Figure 12(1) and Figure 12(2).

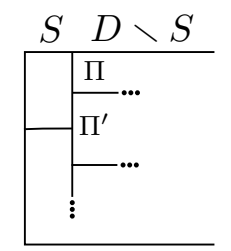

(1) Case 1

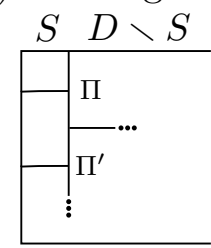

(2) Case 2

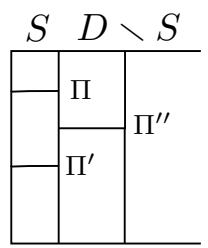

(3) Case 2 - next level

FiguRE 12. Quadrangle with no zipper or extraordinary face.

Since $\Pi$ is not distinguished, the edge shared by $\Pi$ and $\Pi^{\prime}$ does not contain a boundary vertex. In the first case $i\left(\Pi^{\prime}\right) \geqslant 5$, using that $A$ has length at least 4 , contrary to hypothesis. In the second case, $i(\Pi) \geqslant 4$ and $i\left(\Pi^{\prime}\right) \geqslant 4$, so both are ordinary, and we have the situation in Figure 12(3). Let $\Pi^{\prime \prime}$ be the face of $D \backslash S$ adjacent to $\Pi$ and $\Pi^{\prime}$. Either $\Pi^{\prime \prime}$ contains more than one boundary arc, or $\Pi^{\prime \prime}$ is distinguished and contains two distinguished vertices, but both of these are contrary to hypothesis.

3.5. Quadrangle dichotomy. The following proposition says that a combinatorial geodesic quadrangle must be either short, conditions (1) or (2), or thin, (3).

Proposition 3.20. Let $D$ be a simple combinatorial geodesic quadrangle with boundary path $\gamma_{1} \delta_{1} \gamma_{2}^{-1} \delta_{2}^{-1}$. Then one of the following holds:

(1) There exists a face $\Pi$ that intersects both $\gamma_{1}$ and $\gamma_{2}$ in edges with $2=e(\Pi)=i(\Pi)$.

(2) There exist faces $\Pi$ intersecting $\gamma_{1}$ in edges, and $\Pi^{\prime}$ and $\Pi^{\prime \prime}$, both intersecting $\gamma_{2}$ in edges, such that $1=e(\Pi)=e\left(\Pi^{\prime}\right)=e\left(\Pi^{\prime \prime}\right), 4=i(\Pi)=i\left(\Pi^{\prime}\right)=i\left(\Pi^{\prime \prime}\right)$, and any two of $\Pi, \Pi^{\prime}$ and $\Pi^{\prime \prime}$ pairwise intersect in edges. Moreover, $\Pi^{\prime} \cap \Pi^{\prime \prime}$ is an arc connecting $\gamma_{2}$ to $\Pi$, and $\Pi \cap\left(\Pi^{\prime} \cup \Pi^{\prime \prime}\right)$ is connected.

(3) There exist $k \leqslant 6$ and faces $\Pi_{1}, \Pi_{2}, \ldots, \Pi_{k}$, each intersecting $\gamma_{1}$ in edges, such that $\Pi_{1} \cap \delta_{1} \neq \emptyset$ and $\Pi_{k} \cap \delta_{2} \neq \emptyset$ and, for each $1 \leqslant i<k$, we have $\Pi_{i} \cap \Pi_{i+1} \neq \emptyset$. 
The three cases are pictured in Figures 13(1), 13(2), and 13(3), respectively.

Lemma 3.10 implies that in case (3) of Proposition 3.20 the set $\bigcup_{i=1}^{k} \Pi_{i} \cap \gamma_{1}$ is a path subgraph of $\gamma_{1}$.

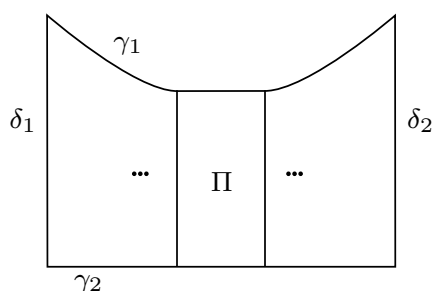

(1) Short (quadrangle)

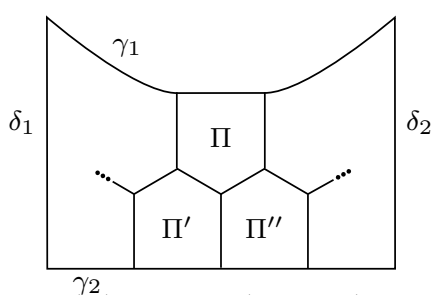

$(2)$ Short (zipper)

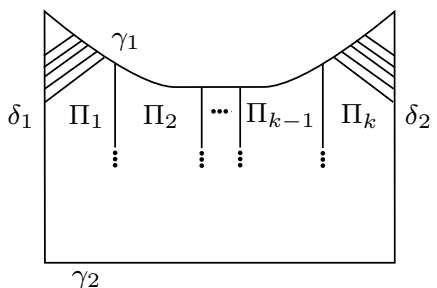

(3) Thin

FiguRE 13. Quadrangle dichotomy

Proof of Proposition 3.20. If $D$ is a single face we are done, so suppose not.

Suppose that the first two conditions do not hold. Let $k>0$ be minimal such that $\Pi_{1}, \ldots, \Pi_{k}$ is a sequence of faces satisfying condition (3). Minimality is equivalent to requiring $\Pi_{i} \cap \delta_{1}=\emptyset$ and $\Pi_{i} \cap \delta_{2}=\emptyset$ for all $1<i<k$.

We may also assume $D$ is non-degenerate, since for triangles we have $k \leqslant 3$, by Theorem 3.13.

Since $D$ is a simple $(3,7)$-diagram it has no valence 1 vertices. If it has non-distinguished valence 2 vertices we can forget them. We then arrange that non-distinguished vertices of $D$ have valence 3 as follows.

Suppose $v$ is a vertex of valence greater than 3. Since $D$ is simple, for any two faces $\Pi$ and $\Pi^{\prime}$ that contain $v$ in their boundaries and do not share an edge incident to $v$, there is a unique way to blow up $v$ to an interior edge $e_{v}$ in such a way that the images of $\Pi$ and $\Pi^{\prime}$ are the faces with $e_{v}$ in their boundaries. Let $\beta$ denote the blow-up map. Since $\Pi$ and $\Pi^{\prime}$ do not share an edge incident to $v$, the two vertices of $e_{v}$ each have valence at least 3 and strictly less than that of $v$.

The only effect of this blow-up on faces is to increase the interior degrees of $\Pi$ and $\Pi^{\prime}$ by 1 each, so $\beta(D)$ is still a simple combinatorial geodesic quadrangle. The faces $\beta\left(\Pi_{1}\right), \ldots, \beta\left(\Pi_{k}\right)$ still satisfy condition $(3)$, since $\beta$ only introduces an interior edge. We now check that $\beta$ does not produce a diagram satisfying conditions (1) or (2) and not condition (3).

We argue for the face $\Pi$. The same arguments apply for $\Pi^{\prime}$. Since $D$ is simple $\Pi$ cannot have $e(\Pi)=2$ and $i(\Pi)=1$, so $\beta(\Pi)$ does not satisfy condition (1).

If $e(\Pi)>1$ then $e(\beta(\Pi))=e(\Pi)>1$, so $\beta(\Pi)$ cannot be one of the faces satisfying condition (2). Suppose now that $e(\Pi)=1$. The blow-up does not change the boundary of $D$, so $\Pi$ is distinguished if and only if $\beta(\Pi)$ is distinguished. If $\Pi$ is distinguished with $i(\Pi) \geqslant 3$ then $\beta(\Pi)$ is distinguished with $i(\beta(\Pi)) \geqslant 4$, so $\beta(D)$ degenerates to a triangle, which implies $k \leqslant 3$, and we are done. Otherwise, if $\Pi$ is distinguished then $i(\beta(\Pi))<4$, so $\beta(\Pi)$ cannot be one of the faces satisfying condition (2). Finally, if $\Pi$ is non-distinguished then the combinatorial geodesic polygon condition requires $i(\Pi) \geqslant 4$, so $i(\beta(\Pi)) \geqslant 5$, and $\beta(\Pi)$ cannot be one of the faces satisfying condition (2).

We conclude that $k \leqslant 3$, in which case we are done, or $\beta(D)$ is a simple non-degenerate combinatorial geodesic quadrangle not satisfying conditions (1) or (2) and containing a sequence $\beta\left(\Pi_{1}\right), \ldots, \beta\left(\Pi_{k}\right)$ of faces satisfying the requirements of condition (3). Moreover, since $\beta$ only introduced an interior edge, $\beta\left(\Pi_{i}\right) \cap \beta\left(\delta_{j}\right)=\emptyset$ for all $1<i<k$ and $j \in\{1,2\}$, so $\beta\left(\Pi_{1}\right), \ldots, \beta\left(\Pi_{k}\right)$ is a minimal length sequence satisfying condition $(3)$ in $\beta(D)$. 
We now repeat blowing up higher valence vertices until either the quadrangle becomes degenerate, and we are done, or there are no higher valence vertices left. Thus, we may assume non-distinguished vertices of $D$ have valence 3 .

If $D$ is irreducible then it is special. If $D$ is special and has no zipper then $k \leqslant 5$, by considering the possibilities given by Theorem 3.18 .

If $D$ is special and has a zipper then, by considering the possible zippered configurations of Lemma 3.16, $k$ is at most four plus the number of teeth of the zipper adjacent to $\gamma_{1}$. Since $D$ does not satisfy condition (2), there can be at most two teeth of the zipper adjacent to $\gamma_{1}$, so $k \leqslant 6$. In fact, $k=6$ can occur, so 6 is the best possible bound for $k$.

The remaining possibility is that $D$ is reducible. It is not vertex reducible, since it is simple. If an edge reduction is possible then so is a face reduction, so suppose $\Pi$ is a face with edges $e, e^{\prime} \subset \partial \Pi$ such that $D$ admits a face reduction at $\left(\Pi, e, e^{\prime}\right)$. Since $D$ is non-degenerate there are four distinguished faces.

Suppose the face reduction separates one distinguished face from the other three, which occurs when one of $e$ or $e^{\prime}$ is an edge of one of the $\gamma_{i}^{\prime}$ 's and the other is an edge of one of the $\delta_{i}$ 's. Then $D$ is a union of a bigon and a quadrangle with fewer faces than $D$. By minimality of $k$, either $\Pi=\Pi_{1}$ or $\Pi=\Pi_{k}$ or $\Pi \neq \Pi_{i}$. Therefore $\Pi_{1}, \ldots, \Pi_{k}$ corresponds to a sequence of faces in the new quadrangle still satisfying condition 3 .

Repeat the argument for the new quadrangle. Since the number of faces in the quadrangle decreases, this process stops after finitely many steps, so we may assume that $D$ is not reducible into a bigon and a quadrangle. Since $D$ is non-degenerate, this implies that every distinguished face has one exterior arc and either 2 or 3 interior arcs, and the distinguished faces are the only ones that intersect both one of the $\delta_{i}$ 's and one of the $\gamma_{i}$ 's.

Suppose there is a face $\Pi$ containing edges $e \subset \partial \Pi \cap \delta_{1}$ and $e^{\prime} \subset \partial \Pi \cap \delta_{2}$. Face reduction at $\left(\Pi, e, e^{\prime}\right)$ sends the $\Pi_{i}$ 's to a minimal length sequence of faces along one side of a combinatorial geodesic triangle connecting the other two sides. Thus, $k \leqslant 3$.

Next, suppose there is an edge $e$ that meets both $\gamma_{1}$ and $\gamma_{2}$. Then $e$ separates $\delta_{1}$ from $\delta_{2}$, so there is some $i<k$ such that $\Pi_{1}, \ldots, \Pi_{i}$ are on one side of $e$ and $\Pi_{i+1}, \ldots, \Pi_{k}$ are on the other. Edge reduction of $D$ at $e$ results in two combinatorial geodesic triangles. In one of these triangles there is a sequence of faces corresponding to $\Pi_{1}, \ldots, \Pi_{i}$ that run along the side corresponding to $\gamma_{1}$ and connect the side corresponding to $\delta_{1}$ to the opposite distinguished vertex. Since we were not in case $(1), i\left(\Pi_{i}\right)>2$, so the face corresponding to $\Pi_{i}$ in the triangle has more than one interior arc. It follows that $i \leqslant 3$. The same argument applied to $\Pi_{i+1}$ shows $k-i \leqslant 3$, so $k \leqslant 6$. (The lower right diagram in Figure 4 shows $k=6$ can be achieved in this case.)

Finally, suppose $D$ has a separating face $\Pi_{i}$ and no separating edge. Face reduction of $D$ at $\Pi_{i}$ yields two combinatorial geodesic triangles. Since $D$ has no separating edge, each of these triangles has a distinguished face corresponding to $\Pi_{i}$ with interior degree greater than one. The same argument as in the previous case then tells us $i \leqslant 3$ and $k-i+1 \leqslant 3$, so $k \leqslant 5$.

\section{Contraction in $G r^{\prime}(1 / 6)$-Groups}

In this section, we study contraction in groups defined by $G r^{\prime}(1 / 6)$-labelled graphs. We give a characterization of strongly contracting geodesics in the Cayley graph in terms of their intersections with embedded components of the defining graph. Throughout this section, $\Gamma$ is an arbitrary $G r^{\prime}(1 / 6)$-labelled graph with a set of labels $\mathcal{S}$, and $X:=\operatorname{Cay}(G(\Gamma), \mathcal{S})$.

Recall that a geodesic $\alpha$ in $X$ is locally $\left(\rho_{1}, \rho_{2}\right)$-contracting if for every embedded component $\Gamma_{0}$ of $\Gamma$ such that $\Gamma_{0} \cap \alpha \neq \emptyset$, closest point projection in $\Gamma_{0}$ of $\Gamma_{0}$ to $\Gamma_{0} \cap \alpha$ is $\left(\rho_{1}, \rho_{2}\right)$-contracting. 
We will prove the following theorem:

Theorem 4.1. A geodesic $\alpha$ in $X$ is locally $\left(\rho_{1}, \rho_{2}\right)$-contracting if and only if there exist $\rho_{1}^{\prime}$ and $\rho_{2}^{\prime}$ such that $\alpha$ is $\left(\rho_{1}^{\prime}, \rho_{2}^{\prime}\right)$-contracting.

Moreover, $\rho_{1}^{\prime}$ and $\rho_{2}^{\prime}$ can be bounded in terms of $\rho_{1}$ and $\rho_{2}$, and when $\rho_{1}(r) \geqslant r / 2$ we can take $\rho_{1}^{\prime}=\rho_{1}$ and $\rho_{2}^{\prime} \asymp \rho_{2}$.

Consequently, $\alpha$ is strongly contracting if and only if it is uniformly locally strongly contracting, $\alpha$ is semi-strongly contracting if and only if it is locally uniformly semi-strongly contracting, and $\alpha$ is sublinearly contracting if and only if it is uniformly locally sublinearly contracting.

Remark 4.2. Theorem 4.1 provides an analogy with the geometry present in many of the preeminent examples of spaces with a mixture of hyperbolic and non-hyperbolic behavior, such as Teichmüller space, the Culler-Vogtmann Outer Space, or relatively hyperbolic spaces. The simplest situation is that geodesics that avoid spending a long time in a non-hyperbolic region behave like hyperbolic geodesics. The analogy here is strongest with relatively hyperbolic spaces, with the embedded components of the defining graph corresponding to the peripheral regions of a relatively hyperbolic space. The peripheral regions are not necessarily non-hyperbolic, so hyperbolic geodesics do not necessarily have to avoid them completely. Rather, a geodesic is roughly as hyperbolic as its intersections with peripheral regions.

Our original motivation for the present paper was to make this analogy precise, and, in particular, to determine whether graphical small cancellation groups contained strongly contracting elements, and therefore fit into the scheme of groups with growth tight actions introduced by Arzhantseva, Cashen, and Tao [8], see Section 5.

In concrete terms, this analogy was first suggested by results of Gruber and Sisto, who proved that the Cayley graph of a graphical small cancellation group is weakly hyperbolic relative to the embedded components of the defining graph [31] in the sense of [19], see also Section 5.2.

Note, however, that in general the groups we consider in the present paper need not be non-trivially relatively hyperbolic $[10,31]$.

4.1. Contraction and Morse quasi-geodesics. We quote some of our technical results from [7] that let us simplify and reformulate the contraction conditions.

Lemma 4.3 ([7, Lemma 6.3]). Let $Y$ and $Y^{\prime}$ be closed subspaces of $X$ at bounded Hausdorff distance from one another. Suppose $Y$ is $\left(r, \rho_{2}\right)$-contracting. Then $Y^{\prime}$ is $\left(r, \rho_{2}^{\prime}\right)$-contracting for some $\rho_{2}^{\prime} \asymp \rho_{2}$. In particular, if $Y$ is strongly contracting then so is $Y^{\prime}$.

Theorem 4.4 ([7, Theorem 1.4, Theorem 7.1]). Let $Y$ be a closed subspace of $X$. Then the following are equivalent:

(1) $Y$ is Morse.

(2) $Y$ is sublinearly contracting.

(3) There exist $\rho_{1}$ and $\rho_{2}$ such that $Y$ is $\left(\rho_{1}, \rho_{2}\right)$-contracting.

(4) There exist a constant $C \geqslant 0$ and a sublinear function $\rho$ such that if $\gamma$ is a geodesic segment then $d(\gamma, Y) \geqslant C$ implies $\operatorname{diam} \pi(\gamma) \leqslant \rho\left(\max _{z \in \gamma} d(z, Y)\right)$.

Moreover, for each implication the function of the conclusion depends only on the function of the hypothesis, not on $Y$.

We remark that in the case $\rho_{1}(r):=r$ the proof of '(3) implies (4)' yields $\rho \asymp \rho_{2}$. When, in addition, $\rho_{2}$ is bounded, which is the strongly contracting case, this recovers the well-known 'Bounded Geodesic Image Property', cf [36, 14]. 
Corollary 4.5. If $Y$ is strongly contracting then every geodesic segment that stays sufficiently far from $Y$ has uniformly bounded projection diameter.

\subsection{Proof of Theorem 4.1.}

Lemma 4.6. Let $\alpha$ be a geodesic in $X$, and let $x$ be a vertex not in $\alpha$. Let $\gamma$ be a path from $x$ to a vertex of $\alpha$ such that $|\gamma|=d(x, \alpha)$. Then, if $p$ is a path from $x$ to a vertex of $\alpha$ such that $p$ is a piece, then $p=\gamma$. In particular, the closest point projection of $p$ to $\alpha$ is $p \cap \alpha$.

Proof. If $p$ has the same terminal vertex as $\gamma$ and if $p \neq \gamma$, then there exist subpaths $\gamma^{\prime}$ of $\gamma$ and $p^{\prime}$ of $p$, each of length at least 1 , such that $c:=\gamma^{\prime} p^{\prime-1}$ is a simple cycle. Since $\gamma^{\prime}$ is a geodesic, we have $\left|\gamma^{\prime}\right| \leqslant|c| / 2$. Since $p^{\prime}$ is a piece we have $\left|p^{\prime}\right|<|c| / 6$. This is a contradiction.

If $p$ has a different terminal vertex, then there exist terminal subpaths $p^{\prime}$ of $p$ and $\gamma^{\prime}$ of $\gamma$, respectively, each having length at least 1, and a path $\alpha^{\prime}$ contained in $\alpha$ such that $c:=\gamma^{\prime} \alpha^{\prime} p^{\prime-1}$ is a simple cycle. By assumption on $\gamma$, we have $\left|\gamma^{\prime}\right| \leqslant\left|p^{\prime}\right|$ and $\left|\alpha^{\prime}\right| \leqslant|c| / 2$, whence we conclude $\left|p^{\prime}\right| \geqslant|c| / 4$, contradicting the fact that $p^{\prime}$ is a piece.

The final claim follows from the fact that a subpath of a piece is a piece.

Lemma 4.7. Let $\Gamma_{0}$ be an embedded component of $\Gamma$ in $X$, and let $\alpha$ be a geodesic in $X$ such that $\Gamma_{0}$ intersects $\alpha$. Closest point projection of $\Gamma_{0}$ to $\alpha$ in $X$ agrees with closest point projection to $\Gamma_{0} \cap \alpha$ in $\Gamma_{0}$.

Proof. Consider a vertex $x \in \Gamma_{0} \backslash \alpha$ such that there exists a point $v \in \alpha$ with $d(x, \alpha)=$ $d(x, v)$ and $v \notin \Gamma_{0}$. Let $\gamma$ be a geodesic from $x$ to $v$, let $p$ be a path in $\Gamma_{0}$ from $x$ to a vertex $v^{\prime}$ of $\alpha$. Let $D$ be a diagram over $\mathcal{R}$ as in Lemma 3.4 filling the triangle $\gamma\left[v, v^{\prime}\right] p^{-1}$, where $\left[v, v^{\prime}\right]$ is the reduced path in $\alpha$ from $v$ to $v^{\prime}$.

Among all possible choices of $x, v, v^{\prime}, \gamma, p$, and $D$ as above, make the choice for which $D$ has the minimal possible number of edges. Note that, by minimality, $D$ is a simple disc diagram.

Let $\Pi$ be a face of $D$ that intersects the side of $D$ corresponding to $p$ in an arc $a$. Then $a$ has a lift to $\Gamma$ via being a subpath of (a copy of) $p$, and one via being a subpath of $\partial \Pi$. If the lifts coincide (up to a label-preserving automorphism of $\Gamma$ ), then we can remove the edges of $a$ from $D$, thus obtaining a path $p^{\prime}$ in $\Gamma_{0}$ as above, contradicting minimality. Hence, $a$ is a piece. Therefore, Lemma 4.6 implies that the side of $D$ corresponding to $p$ is not contained in a single face of $D$.

We make a diagram $D^{\prime}$ by attaching a new face $\Pi^{\prime}$ to $D$ by identifying a proper subpath of the boundary of $\Pi^{\prime}$ with the side of $D$ corresponding to $p$. This operation is purely combinatorial, the boundary of $\Pi^{\prime}$ is not labelled. Note that if $\Pi$ is a face of $D$ with $e(\Pi)=1$ whose exterior arc is contained in $p$ then, by the previous paragraph, that exterior arc is a piece. Since interior arcs of $D$ are pieces, $i(\Pi) \geqslant 6$ in $D$. Thus, $\Pi$ becomes an interior face of $D^{\prime}$ with $i(\Pi) \geqslant 7$. It follows that $D^{\prime}$ is a combinatorial geodesic bigon.

Apply Theorem 3.13 to $D^{\prime}$ : it has at most two distinguished faces, one is $\Pi^{\prime}$ and the other, if it exists, is at the vertex corresponding to $v$. Any other face of $D^{\prime}$ came from $D$, and, in particular, the side of $D$ corresponding to $p$ is contained in a single face of $D$. This is a contradiction.

Lemma 4.7 immediately implies the global-to-local direction of Theorem 4.1.

Lemma 4.8. Let $\alpha$ be a geodesic in $X$. Let $p_{1} p_{2}$ be a simple path starting at a vertex $y$ in $\alpha$ and terminating at a vertex $x \in X$ such that $p_{1} p_{2}$ is contained in some embedded component $\Gamma_{0}$ and such that each $p_{i}$ is a piece that is not a single vertex. Then $d(x, \alpha)>$ $\max \left\{\left|p_{1}\right|,\left|p_{2}\right|\right\}$. 
Proof. Let $q$ be a path starting at $x$ and terminating at a vertex $y^{\prime}$ in $\alpha$ such that $|q|=d(x, \alpha)$. If $y^{\prime}=y$, then the claim follows from the convexity of $\Gamma_{0}$, noting that any simple path that is a concatenation of two pieces must be a geodesic. Similarly, if $q \cap p_{1} \neq \emptyset$ then Lemma 4.6 implies $q=p_{1} p_{2}$. Hence, assume that $q \cap p_{1}=\emptyset$.

Without loss of generality, we may assume $q \cap p_{2}=x$. Consider a diagram $D$ over $\mathcal{R}$ as in Lemma 3.4 filling the embedded geodesic quadrangle $p_{1} p_{2} q\left[y^{\prime}, y\right]$. We may stick onto the 2-complex $D$ two 2-cells $\Pi_{1}$ and $\Pi_{2}$ by identifying proper subpaths of their boundaries with $p_{1}$ and $p_{2}$, respectively. By construction, this yields a combinatorial geodesic triangle, and by Theorem 3.13, it has shape $I_{1} I_{1}$, where $\Pi_{1}$ and $\Pi_{2}$ are the distinguished faces intersecting each other only in a valence 4 vertex. Using Lemma 4.6, there are no faces with exterior degree 2 , so $D$ is a single face.

This shows that there exists a simple cycle of the form $c:=p_{1} p_{2} q\left[y^{\prime}, y\right]$ in some embedded component $\Gamma_{1}$. Since $\left|p_{1}\right|,\left|p_{2}\right|<|c| / 6$ and $\left|\left[y^{\prime}, y\right]\right| \leqslant|c| / 2$, we have $|q|>|c| / 6>$ $\max \left\{\left|p_{1}\right|,\left|p_{2}\right|\right\}$.

Lemma 4.9. Let $\alpha$ be a geodesic in $X$. Let $\Gamma_{0}$ be an embedded component of $\Gamma$ intersecting $\alpha$. Suppose $\alpha \cap \Gamma_{0}$ is $\left(\rho_{1}, \rho_{2}\right)$-contracting in $\Gamma_{0}$. Let $c$ be a simple cycle in $\Gamma_{0}$ such that $c=p_{1} p_{2} q a$, where each $p_{i}$ is a piece, $q$ realizes the distance of the terminal vertex $x$ of $p_{2}$ to $\alpha$, and $a$ is a subpath of $\alpha$. Then $|c|$ is bounded, with bound depending only on $\rho_{1}$ and $\rho_{2}$.

Proof. Let $x:=p_{2} \cap q$, and let $y:=p_{2} \cap p_{1}$. Since any subpath of a piece is a piece itself, we may assume without loss of generality that $x$ is the point of $p_{2}$ maximizing $\operatorname{diam} \pi(x) \cup \pi(y)$, where $\pi$ denotes closest point projection to $\alpha$ in $X$. By Lemma 4.6, we have $|a| \leqslant \operatorname{diam} \pi(x) \cup \pi(y)$.

Since $p_{1} p_{2}$ is a path from $\alpha$ to $x$ and $q$ minimizes distance, $|q| \leqslant\left|p_{1}\right|+\left|p_{2}\right|<|c| / 3$, which implies $|a|=|c|-\left|p_{1}\right|-\left|p_{2}\right|-|q|>|c| / 3$.

Let $C \geqslant 0$ be as Theorem 4.4 (4). If $\left|p_{1}\right|<C$, then $|q| \leqslant\left|p_{1}\right|+\left|p_{2}\right|<C+|c| / 6$, which implies $|c|<12 C$.

Otherwise, Lemma 4.8 implies that for every point $z \in p_{2}$ we have $d(z, \alpha) \geqslant C$, so $p_{2}$ is a geodesic that stays outside the $C$-neighborhood of $\alpha$. Theorem 4.4 (4) says $\operatorname{diam} \pi\left(p_{2}\right)$ is bounded by a sublinear function of $\max _{z \in p_{2}} d(z, \alpha)<|c| / 3$. Thus, $|c| / 3<|a| \leqslant \operatorname{diam} \pi\left(p_{2}\right)$ is bounded above by a sublinear function of $|c|$, which implies $|c|$ is bounded.

Lemma 4.10. Let $\alpha$ be a geodesic in $X$. Let $\Gamma_{0}$ be an embedded component of $\Gamma$ intersecting $\alpha$. Suppose $\alpha \cap \Gamma_{0}$ is $\left(\rho_{1}, \rho_{2}\right)$-contracting in $\Gamma_{0}$. Let $c$ be a simple cycle in $\Gamma_{0}$ of the form $c=p_{1} p_{2} p_{3} p_{4} a$, where each $p_{i}$ is a piece and $a$ is a subpath of $\alpha$. Then $|c|$ is bounded, with bound depending only on $\rho_{1}$ and $\rho_{2}$.

Proof. Let $x:=p_{2} \cap p_{3}$. Let $y_{1}:=p_{1} \cap \alpha$. Let $y_{2}:=p_{4} \cap \alpha$. By Lemma 4.7, there is a path $q \subset \Gamma_{0}$ such that $|q|=d(x, \alpha)$. Let $y^{\prime}:=q \cap \alpha$.

By symmetry, we may suppose $q \cap p_{3}=x$. Apply Lemma 4.9 to see that $|q|+\left|p_{3}\right|+$ $\left|p_{4}\right|+d\left(y_{2}, y^{\prime}\right)$ is uniformly bounded. If $q$ coincides with $p_{1} p_{2}$ we are done. Otherwise it must be that $q \cap p_{1}=\emptyset$, for otherwise we would have a simple cycle composed of a geodesic and one or two pieces, which is impossible. Let $p_{2}^{\prime}:=\overline{p_{2} \backslash q}$, and let $q^{\prime}:=\overline{q \backslash p_{2}}$. Apply Lemma 4.9 to see $\left|q^{\prime}\right|+\left|p_{2}^{\prime}\right|+\left|p_{1}\right|+d\left(y_{1}, y^{\prime}\right)$ is uniformly bounded. Thus, $|c|$ is uniformly bounded.

Lemma 4.11. Let $\alpha$ be a geodesic in $X$. Let $\Gamma_{0}$ be an embedded component of $\Gamma$ intersecting $\alpha$. Suppose $\alpha \cap \Gamma_{0}$ is $\left(\rho_{1}, \rho_{2}\right)$-contracting in $\Gamma_{0}$. Let $c$ be a simple cycle in $\Gamma_{0}$ such that $c=q_{1} p q_{3} a$, where $p$ is a piece, $q_{1}$ and $q_{3}$ are geodesics realizing the closest point projections of the endpoints of $p$ to $\alpha$, and $a$ is a subpath of $\alpha$. Then there is a sublinear function $\rho_{2}^{\prime}$ 
depending only on $\rho_{1}$ and $\rho_{2}$ such that $\operatorname{diam} \pi(c) \leqslant \rho_{2}^{\prime}(|c|)$. If $\rho_{1}(r) \geqslant r / 2$ then we can take $\rho_{2}^{\prime}:=2 \rho_{2}$.

Proof. Among all path subgraphs of $p$, consider those with maximal projection diameter. Among those, choose one, $p^{\prime}$, with minimal length, and let $x^{\prime}$ and $y^{\prime}$ be its endpoints. Let $x^{\prime \prime} \in \pi\left(x^{\prime}\right)$ and $y^{\prime \prime} \in \pi\left(y^{\prime}\right)$ be vertices such that $d\left(x^{\prime \prime}, y^{\prime \prime}\right)=\operatorname{diam} \pi\left(x^{\prime}\right) \cup \pi\left(y^{\prime}\right)$. By Lemma 4.7 there are geodesics $q_{1}^{\prime} \subset \Gamma_{0}$ connecting $x^{\prime}$ to $x^{\prime \prime}$ and $q_{3}^{\prime} \subset \Gamma_{0}$ connecting $y^{\prime}$ to $y^{\prime \prime}$. Let $a^{\prime}$ be the subpath of $\alpha$ from $x^{\prime \prime}$ to $y^{\prime \prime}$. Let $c^{\prime}:=q_{1}^{\prime} p^{\prime} q_{3}^{\prime} a^{\prime}$. The maximality hypothesis on $p^{\prime}$ implies $\pi\left(p^{\prime}\right) \subset a^{\prime}$ and diam $\pi\left(p^{\prime}\right)=\operatorname{diam} \pi(p)$. It is immediate from the definitions that $\operatorname{diam} \pi(c)=\operatorname{diam} \pi(p)$, so it suffices to bound $d\left(x^{\prime \prime}, y^{\prime \prime}\right)$.

Let $x$ and $y$ be the endpoints of $p$, and note that $\left|q_{1}^{\prime}\right|+\left|q_{3}^{\prime}\right| \leqslant\left|q_{1}\right|+d\left(x, x^{\prime}\right)+d\left(y, y^{\prime}\right)+$ $\left|q_{3}\right| \leqslant|c|$. If there exists a vertex $z \in q_{1}^{\prime} \cap q_{3}^{\prime}$ then both $x^{\prime \prime}$ and $y^{\prime \prime}$ are in $\pi(z)$, so $d\left(x^{\prime \prime}, y^{\prime \prime}\right) \leqslant \rho_{2}(d(z, \alpha)) \leqslant \rho_{2}\left(\left|q_{1}^{\prime}\right|\right) \leqslant \rho_{2}(|c|)$, and we are done. Otherwise, minimality of $\left|p^{\prime}\right|$ implies $c^{\prime}$ is a simple cycle.

Since $p^{\prime}$ is a piece and $a^{\prime}$ is geodesic, we have $\left|q_{1}^{\prime}\right|+\left|q_{3}^{\prime}\right|>\left|c^{\prime}\right| / 3>2\left|p^{\prime}\right|$, so there exists a point $z^{\prime} \in p^{\prime}$ such that $d\left(x^{\prime}, z^{\prime}\right) \leqslant\left|q_{1}^{\prime}\right| / 2$ and $d\left(z^{\prime}, y^{\prime}\right) \leqslant\left|q_{3}^{\prime}\right| / 2$.

If $\rho_{1}(r) \geqslant r / 2$, then take $\rho_{1}^{\prime}:=\rho_{1}$ and $\rho_{2}^{\prime \prime}:=\rho_{2}$. Otherwise, by Theorem $4.4(2)$ there exists a sublinear function $\rho_{2}^{\prime \prime}$ such that $\alpha \cap \Gamma_{0}$ is $\left(\rho_{1}^{\prime}, \rho_{2}^{\prime \prime}\right)$-contracting in $\Gamma_{0}$ for $\rho_{1}^{\prime}(r):=r$. Let $\rho_{2}^{\prime}:=2 \rho_{2}^{\prime \prime}$. Since $\pi\left(p^{\prime}\right) \subset a^{\prime}$, we conclude:

$$
\begin{aligned}
d\left(x^{\prime \prime}, y^{\prime \prime}\right) & \leqslant \operatorname{diam} \pi\left(x^{\prime}\right) \cup \pi\left(z^{\prime}\right)+\operatorname{diam} \pi\left(y^{\prime}\right) \cup \pi\left(z^{\prime}\right) \\
& \leqslant \rho_{2}^{\prime \prime}\left(d\left(x^{\prime}, \alpha\right)\right)+\rho_{2}^{\prime \prime}\left(d\left(y^{\prime}, \alpha\right)\right) \\
& =\rho_{2}^{\prime \prime}\left(\left|q_{1}^{\prime}\right|\right)+\rho_{2}^{\prime \prime}\left(\left|q_{3}^{\prime}\right|\right) \leqslant 2 \rho_{2}^{\prime \prime}(|c|)=\rho_{2}^{\prime}(|c|)
\end{aligned}
$$

Lemma 4.12. Let $\alpha$ be a locally $\left(\rho_{1}, \rho_{2}\right)$-contracting geodesic in $X$. Let $Y \subset X$ be either an embedded component of $\Gamma$, a piece, or a single vertex. Then there is a sublinear function $\rho_{2}^{\prime}$ depending only on $\rho_{1}$ and $\rho_{2}$ such that if $Y$ is disjoint from $\alpha$ then $\operatorname{diam} \pi(Y) \leqslant \rho_{2}^{\prime}(d(Y, \alpha))$. If $\rho_{1}(r) \geqslant r / 2$ we can take $\rho_{2}^{\prime} \asymp \rho_{2}$.

Proof. Suppose $Y$ is disjoint from $\alpha$ and choose a vertex $y \in Y$ such that $d(y, \alpha)=d(Y, \alpha)$. Let $y^{\prime}$ be a point in $\pi(y)$. It suffices to show that there exists a sublinear function $\rho_{2}^{\prime \prime}$ such that for every $x^{\prime} \in \pi(Y)$ we have $d\left(x^{\prime}, y^{\prime}\right) \leqslant \rho_{2}^{\prime \prime}(d(Y, \alpha))$. Given such a $\rho_{2}^{\prime \prime}$, set $\rho_{2}^{\prime}:=2 \rho_{2}^{\prime \prime}$, and the lemma follows from the triangle inequality.

If $\pi(Y)=\left\{y^{\prime}\right\}$ we are done. Otherwise, let $x^{\prime}$ be an arbitrary point in $\pi(Y) \backslash\left\{y^{\prime}\right\}$. Let $\alpha^{\prime}$ be the subpath of $\alpha$ from $x^{\prime}$ to $y^{\prime}$.

Choose $x \in Y$ such that $x^{\prime} \in \pi(x)$. Choose a path $p$ from $x$ to $y$ in $Y$, and geodesics $\beta_{1}$ and $\beta_{2}$ connecting $x$ to $x^{\prime}$ and $y$ to $y^{\prime}$, respectively. Choose a diagram $D$ over $\mathcal{R}$ as in Lemma 3.4 filling $\alpha^{\prime} \beta_{2}^{-1} p^{-1} \beta_{1}$. Assume that we have chosen $x, p, \beta_{1}, \beta_{2}$, and $D$ so that $D$ has the minimal number of edges among all possible choices.

In the case that $\beta_{1}$ and $\beta_{2}$ intersect, let $D^{\prime}$ be the disc component of $D$ intersecting the side corresponding to $\alpha$. Then $D^{\prime}$ is a combinatorial geodesic triangle. Apply Theorem 3.13 to $D^{\prime}$. Since $\beta_{1}$ and $\beta_{2}$ are geodesics realizing closest point projection, the only possibilities are that $D^{\prime}$ is:

(1) a single face,

(2) shape $I_{2}$, where $\alpha^{\prime}$ is the side joining two vertices in the same distinguished face,

(3) shape IV with exactly three faces incident to $\alpha^{\prime}$, two corners with interior degree 2 each and one ordinary face, or

(4) shape $\mathrm{V}$ with exactly two faces incident to $\alpha^{\prime}$, the two corners with interior degree 2 each.

In all cases, $d\left(x^{\prime}, y^{\prime}\right)$ is bounded by a sublinear function $\rho_{2}^{\prime \prime}$ of $d(Y, \alpha)$ that depends only on $\rho_{1}$ and $\rho_{2}$ : For case (1) this follows from the fact that $\alpha$ is uniformly locally contracting. 
For case (3) this follows from Lemma 4.9 and Lemma 4.10, and for case (4) this follows from Lemma 4.9. In these two cases, the bounds are in fact constants depending only on $\rho_{1}$ and $\rho_{2}$. Now consider case (2). Let $\Pi$ be the face of $D^{\prime}$ containing $\alpha^{\prime}$. Let $c$ be the embedded quadrangle in $X$ whose sides are $\alpha^{\prime}$, a subpath $q_{1}$ of $\beta_{1}$, a piece $p^{\prime}$, and a subpath $q_{2}$ of $\beta_{2}$. Apply Lemma 4.11 to $c$, and observe that ||$q_{1}|-| q_{2}|| \leqslant\left|p^{\prime}\right|<|c| / 6$, and $\left|q_{1}\right|+\left|q_{2}\right|>|c| / 3$, whence $d(Y, \alpha)=\left|\beta_{2}\right| \geqslant\left|q_{2}\right|>|c| / 6$.

Now suppose that $\beta_{1}$ and $\beta_{2}$ do not intersect. In this case minimality of $D$ and the fact that $y$ minimizes the distance from $Y$ to $\alpha$ imply that $D$ is simple. If $Y$ is an embedded component of $\Gamma$, it follows as in the proof of Lemma 4.7 that any arc in the side of $D$ corresponding to $p$ is a piece. The same is true if $Y$ is a piece since subpaths of pieces are pieces. Thus, we can stick a new face onto $p$ to obtain a combinatorial geodesic triangle $D^{\prime}$, and we make the same argument as above, noting this time that case (1) cannot hold, for it would imply that $Y$ intersects $\alpha$.

Proof of Theorem 4.1. Recall that the global-to-local direction of Theorem 4.1 follows from Lemma 4.7.

Suppose that $\alpha$ is locally $\left(\rho_{1}, \rho_{2}\right)$-contracting.

Let $x$ and $y$ be points of $X$ such that $d(x, y) \leqslant \rho_{1}(d(x, \alpha))$. Let $\gamma$ be a geodesic from $x$ to $y$. Let $x^{\prime} \in \pi(x)$ and $y^{\prime} \in \pi(y)$ be points realizing diam $\pi(x) \cup \pi(y)$. Let $\delta_{1}$ be a geodesic from $x^{\prime}$ to $x$, and let $\delta_{2}$ be a geodesic from $y^{\prime}$ to $y$. Let $\alpha^{\prime}$ be the path subgraph of $\alpha$ from $x^{\prime}$ to $y^{\prime}$.

First, assume that $\alpha^{\prime}$ does not enter the $C$-neighborhood of $\gamma$, where $C$ is the constant from Theorem 4.4 (4) associated to $\left(\rho_{1}, \rho_{2}\right)$.

If $\delta_{1}$ and $\delta_{2}$ intersect, Lemma 4.12 yields the claim, whence we will assume that they do not. Moreover, by removing initial and terminal subpaths of $\gamma$ that do not increase the size of the closest point projection to $\alpha$, we may assume that $\gamma, \delta_{1}, \delta_{2}$ and $\alpha^{\prime}$ can be concatenated to a simple closed path $c_{0}$. Let $D$ be a diagram as in Lemma 3.4 for the label of $c_{0}$, and, by identifying $\partial D$ with $c_{0}$, we can consider $\gamma, \delta_{1}, \delta_{2}$ and $\alpha^{\prime}$ as subpaths of $\partial D$. As the interior arcs of $D$ are pieces and its four sides are geodesics, we can apply Proposition 3.20.

The first possibility is that there is a face $\Pi$ with $e(\Pi)=2$ and $i(\Pi)=2$. Its boundary is a cycle $c=p_{1} q p_{3} a$ in some embedded component such that the $p_{i}$ are pieces, $a$ is a path subgraph of $\alpha$, and $q$ is a path subgraph of $\gamma$. We have $|c| / 6<|a| \leqslant|c| / 2$. Therefore, $\max _{z \in c} d(z, \alpha) \leqslant 5|c| / 12$

We now choose $R_{2} \geqslant 0$ so that for all $r \geqslant R_{2}$ we have $2 \rho_{2}(r)<\rho_{1}(r)$. Suppose $\gamma$ does not enter the $R_{2}$-neighborhood of $\alpha$.

By Theorem 4.4 (4) we have that $|c| / 6<|a|$ is bounded by a sublinear function of $\max _{z \in c} d(z, \alpha) \leqslant 5|c| / 12$. This implies $|c|$ is uniformly bounded, as are $\left|p_{1}\right|,\left|p_{3}\right|<|c| / 6$. Therefore, $\gamma$ enters a uniformly bounded neighborhood of $\alpha$.

The second possibility is that $D$ contains a zipper with two teeth on $\alpha$. By Lemma 4.10, the boundary lengths of the two teeth on $\alpha$ are uniformly bounded. It follows that the boundary length of the upper tooth is also uniformly bounded, since its intersection with the bottom teeth accounts for more than $1 / 6$-th of its length. Therefore, $\gamma$ enters a uniformly bounded neighborhood of $\alpha$.

Let $C^{\prime}$ be larger than $C, R_{2}$, and the bounds from the first two cases. Let $z$ be the first point of $\gamma$ such that $d(z, \alpha) \leqslant C^{\prime}$, if such a point exists. Otherwise let $z:=y$. The projection diameter of $\gamma$ is at most the projection diameter of the path subgraph of $\gamma$ from $x$ to $z$ plus the projection diameter of the path subgraph from $z$ to $y$. The latter is at most $4 C^{\prime}$, since every point of this path is within $2 C^{\prime}$ of $\alpha$, so it suffices to bound 
the former. Thus, we may assume that the geodesic from $x$ to $y$ does not enter the $C^{\prime}$ neighborhood of $\alpha$.

By our choice of $C^{\prime}$, we conclude that $D$ must fall into the third case of Proposition 3.20. Therefore, there exist $k \leqslant 6$ and a path graph $p:=p_{0} p_{1} p_{2} \ldots p_{k} p_{k+1}$ (recall Lemma 3.10) from $\delta_{1}$ to $\delta_{2}$ such that:

- If $1 \leqslant i \leqslant k$, then $p_{i}$ is a path subgraph of $\gamma \cap \Pi_{i}$, and

- $p_{0}$ is empty or a piece in $\Pi_{1}$, and $p_{k+1}$ is empty or a piece in $\Pi_{k}$.

The second claim follows since, in $D$, the corner that is separated from the rest of $D$ by removing $\Pi_{1}$ is either empty, a face, or has shape $\mathrm{I}_{1}$. The same observation holds for the corner at $\Pi_{k}$.

Notice that every point of $\gamma$ is within $d(x, \alpha)+\rho_{1}(d(x, \alpha)) \leqslant 2 d(x, \alpha)$ of $\alpha$.

For $1 \leqslant i \leqslant k$, the path graph $p_{i}$ is a geodesic subsegment of $\gamma$ that is outside the $R_{2}$-neighborhood of $\alpha$. If $p_{i}$ is contained in an embedded component $\Gamma_{0}$ of $\Gamma$ disjoint from $\alpha$, then diam $\pi\left(p_{i}\right)$ is bounded by a sublinear function of $d\left(\Gamma_{0}, \alpha\right) \leqslant d\left(p_{i}, \alpha\right)<2 d(x, \alpha)$, by Lemma 4.12. If $p_{i}$ is not contained in an embedded component disjoint from $\alpha$, then Theorem 4.4 (4) says diam $\pi\left(p_{i}\right)$ is bounded by a sublinear function of $\max _{z \in p_{i}} d(z, \alpha) \leqslant$ $2 d(x, \alpha)$. When $i \in\{0, k+1\}$, the diameter of $\pi\left(p_{i}\right)$ is bounded by a sublinear function of $d\left(p_{i}, \alpha\right)$ by Lemma 4.12 .

We have that $\operatorname{diam} \pi(x) \cup \pi(y) \leqslant \sum_{i=0}^{k+1} \operatorname{diam} \pi\left(p_{i}\right)$, and each of the at most 8 terms is bounded by a sublinear function of $d(x, \alpha)$, so diam $\pi(x) \cup \pi(y)$ is bounded by a sublinear function $\rho_{2}^{\prime}$ of $d(x, \alpha)$. Thus, $\alpha$ is $\left(r, \rho_{2}^{\prime}\right)$-contracting. Moreover, each of the constituent sublinear functions of $\rho_{2}^{\prime}$ is determined by $\rho_{1}$ and $\rho_{2}$ and, when $\rho_{1}(r) \geqslant r / 2$, is either bounded or asymptotic to $\rho_{2}$, so $\rho_{2}^{\prime}$ depends only on $\rho_{1}$ and $\rho_{2}$, and we can take $\rho_{2}^{\prime} \asymp \rho_{2}$ when $\rho_{1}(r) \geqslant r / 2$.

\subsection{First applications of Theorem 4.1. Theorem 4.1 and Theorem 4.4 show:}

Theorem 4.13. A geodesic in $X$ is Morse if and only if it is uniformly locally contracting.

In the classical small cancellation case we have more explicit criteria:

Corollary 4.14. Let $\Gamma$ be a $G r^{\prime}(1 / 6)$-labelled graph whose components are cycle graphs. Let $\alpha$ be a geodesic in $X$. Define $\rho(r):=\max _{\left|\Gamma_{i}\right| \leqslant r}\left|\Gamma_{i} \cap \alpha\right|$, where the $\Gamma_{i}$ range over embedded components of $\Gamma$. Then $\alpha$ is Morse if and only if $\rho$ is sublinear, and $\alpha$ is strongly contracting if and only if $\rho$ is bounded.

In hyperbolic spaces and in $\mathrm{CAT}(0)$ spaces Morse geodesics are known to be strongly contracting. In graphical small cancellation groups we build the first examples with a wide range of degrees of contraction:

Theorem 4.15. Let $\rho$ be a sublinear function. There exists a group $G$ with finite generating set $\mathcal{S}$ and a function $\rho^{\prime} \asymp \rho$ such that there exists an $\left(r, \rho^{\prime}\right)$-contracting geodesic $\alpha$ in the Cayley graph $X$ of $G$ with respect to $\mathcal{S}$.

Furthermore, $\rho^{\prime}$ is optimal, in the following sense: If $\alpha$ is $\left(r, \rho^{\prime \prime}\right)$-contracting for some $\rho^{\prime \prime}$ then $\limsup _{r \rightarrow \infty} \frac{\rho^{\prime \prime}(2 r)}{\rho(r)} \geqslant 1$.

If $\lambda>0$, a $C^{\prime}(\lambda)$-collection of words $W$ is a subset of $\langle\mathcal{S}\rangle$ such that the disjoint union of cycle graphs labelled by the elements of $W$ satisfies the graphical $C^{\prime}(\lambda)$-condition.

Proof. We may assume $\rho$ is unbounded and integer valued. Since $\rho$ is sublinear, there exists an $R$ such that $5 \rho(r) \leqslant 2 r$ for all $r \geqslant R$. Let $\mathcal{S}:=\{a, b, c\}$. Let $I \subset\{z \in \mathbb{Z} \mid z \geqslant R\}$ be an infinite set such that there exists a $C^{\prime}(1 / 12)$-collection $\left\{w_{i}\right\}_{i \in I}$ of words $w_{i} \in\langle b, c\rangle$ with $\left|w_{i}\right|=4 i$. For $i \in I$, define $R_{i}:=a^{\rho(i)} w_{i}$. 
Let $\Gamma:=\left(\Gamma_{i}\right)_{i \in I}$ be a disjoint union of $\mathcal{S}$-labelled cycle graphs, with $\Gamma_{i}$ labelled by $R_{i}$. Let $G:=G(\Gamma)$ and $X:=\operatorname{Cay}(G, \mathcal{S})$. There are no non-trivial label-preserving automorphisms of any component $\Gamma_{i}$ because of the unique $a$-labelled path subgraph. There are no non-trivial label-preserving automorphisms of $\Gamma$ that exchange components since $\left\{w_{i}\right\}_{i \in I}$ have distinct lengths.

If $p$ is a piece contained in $\Gamma_{i}$ and labelled by $l$ then $l$ can be written $l=l^{\prime}+l^{\prime \prime}+l^{\prime \prime \prime}$ where $l^{\prime}$ is a suffix of $w_{i}, l^{\prime \prime}$ is a subword of $a^{\rho(i)}$, and $l^{\prime \prime \prime}$ is a prefix of $w_{i}$. If $l^{\prime}$ or $l^{\prime \prime \prime}$ is non-empty then $l^{\prime} l^{\prime \prime \prime}$ is a piece for $\left\{w_{i}\right\}_{i \in I}$. Therefore $|p|<\rho(i)+\left|w_{i}\right| / 12$. Since $5 \rho(i) \leqslant 2 i$ this implies $|p|<\left|R_{i}\right| / 6$, so $\Gamma$ is $C^{\prime}(1 / 6)$-labelled.

Let $\alpha$ be the geodesic with all edge labels $a$. By construction, $\alpha$ is locally $(r, \rho)-$ contracting, so Theorem 4.1 says there exists $\rho^{\prime} \asymp \rho$ such that $\alpha$ is $\left(r, \rho^{\prime}\right)$-contracting.

Conversely, if $\alpha$ is $\left(r, \rho^{\prime \prime}\right)$-contracting then it is locally $\left(r, \rho^{\prime \prime}\right)$-contracting. By construction, for $i \in I$ there exists a point $x_{i}$ such that $d\left(x_{i}, \alpha\right)=2 i$ and $\operatorname{diam} \pi\left(x_{i}\right)=\rho(i)$, so we must have $\rho(i) \leqslant \rho^{\prime \prime}(2 i)$.

Theorem 4.16 shows that in classical small cancellation groups the geometry of cycle graphs dictates that only the output contraction function $\rho_{2}$ plays a role. In Theorem 4.19 we construct a graphical example for which the input contraction function $\rho_{1}$ also carries non-trivial information.

Theorem 4.16. Let $\Gamma$ be a $G r^{\prime}(1 / 6)$-labelled graph whose components are cycle graphs. A geodesic $\alpha$ in $X$ that is $\left(\rho_{1}, \rho_{2}\right)$-contracting is $\left(r, \rho_{2}^{\prime}\right)$-contracting for $\rho_{2}^{\prime} \asymp \rho_{2}$.

Proof. Let $\Gamma_{0}$ be an embedded component of $\Gamma$. Since $\Gamma_{0}$ is a cycle graph and $\Gamma_{0} \cap \alpha$ is connected, there is a unique point $y$ for which the closest point projection $\pi(y)$ in $\Gamma_{0}$ has positive diameter, and $\operatorname{diam} \pi(y)=\operatorname{diam} \pi\left(\Gamma_{0} \cap \alpha\right) \leqslant \rho_{2}(d(y, \alpha))$.

Let $x$ be a point of $\Gamma_{0}$ equidistant from $y$ and $\alpha$. Then $d(x, y)=d(x, \alpha)$ and:

$$
\operatorname{diam} \pi(x) \cup \pi(y)=\operatorname{diam} \pi(y) \leqslant \rho_{2}(d(y, \alpha))=\rho_{2}(2 d(x, \alpha))=\rho_{2}^{\prime}(d(x, \alpha))
$$

This is the worst case, since for $x^{\prime}$ closer to $\alpha$ the ball of radius $d\left(x^{\prime}, \alpha\right)$ about $x^{\prime}$ does not include $y$. We conclude that $\alpha$ is locally $\left(r, \rho_{2}(2 r)\right)$-contracting. Now apply Theorem 4.1 to see that $\alpha$ is $\left(r, \rho_{2}^{\prime}\right)$-contracting for some $\rho_{2}^{\prime}(r) \asymp \rho_{2}(2 r) \asymp \rho_{2}(r)$.

Corollary 4.17. Let $\Gamma$ be a $G r^{\prime}(1 / 6)$-labelled graph whose components are cycle graphs. A geodesic $\alpha$ in $X$ that is $\left(\rho_{1}, \rho_{2}\right)$-contracting with $\rho_{2}$ bounded is strongly contracting.

Having uniformly bounded intersection with every embedded component of $\Gamma$ is a sufficient condition for a geodesic $\alpha$ in the Cayley graph of a $G r^{\prime}(1 / 6)$-group to be strongly contracting. It is not a necessary condition. Consider the following example:

Example 4.18. We start with a simple example of an infinite classical $C^{\prime}(1 / 6)$-small cancellation presentation: $\left\langle a, b \mid R_{n}: n \in \mathbb{N}\right\rangle$ where, for each $n \geqslant 0$ we define:

$$
R_{n}=a b^{20 n+1} a b^{20 n+2} \cdots a b^{20 n+19} a b^{-(20 n+20)}
$$

The graphs $\Gamma_{i}$ for $i \geqslant 1$ are defined by taking the disjoint union of oriented cycles $R_{n}$ for $(i-1)(i) \leqslant 2 n \leqslant-2+i(i+1)$ and identifying the unique subpath with label $b^{20 k}$ in $R_{k-1}$ to the unique subpath in $R_{k}$ with label $b^{20 k}$ which is preceded by $a$ and succeeded by $b a$. This means we identify the paths labelled by the bold words $a b^{20(k-1)+1} a b^{20(k-1)+2} \ldots a \mathbf{b}^{-\mathbf{2 0 k}}$ and $a \mathbf{b}^{\mathbf{2 0 k}} b a \ldots a b^{-(20 k+20)}$.

The $\{a, b\}$-labelled graph $\sqcup_{i \in \mathbb{N}} \Gamma_{i}$ satisfies the $G r^{\prime}(1 / 6)$-condition and gives rise to the same Cayley graph as $\left\langle a, b \mid R_{n}: n \in \mathbb{N}\right\rangle$. Consider a path $\alpha$ in the Cayley graph labelled by the powers of $a$. For every $i$, there exists an embedded copy $\Gamma_{i}^{\prime}$ of $\Gamma_{i}$ with $\left|\alpha \cap \Gamma_{i}^{\prime}\right|=i$. Since paths in $\Gamma_{i}$ labelled by powers of $a$ are geodesic, this implies that $\alpha$ is a geodesic. It 

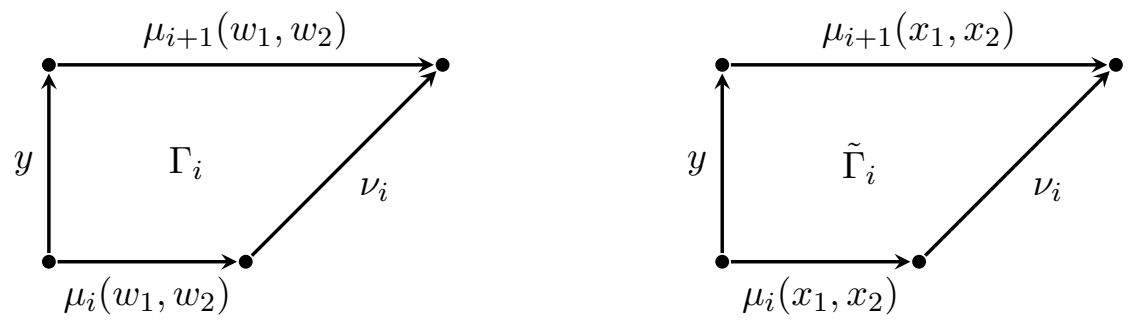

FiguRE 14. The graphs $\Gamma_{i}$ and $\tilde{\Gamma}_{i}$.

has intersection of length at most 1 with relators $R_{n}$, so it is strongly contracting, but we have $\left|\alpha \cap \Gamma_{i}^{\prime}\right|=i$.

We also provide the first examples spaces $X$ and $\tilde{X}$ and geodesics $\gamma$ and $\tilde{\gamma}$ such that there exists a quasi-isometry $X \rightarrow \tilde{X}$ mapping $\gamma$ to $\tilde{\gamma}$ and such that $\gamma$ is not strongly contracting, but $\tilde{\gamma}$ is strongly contracting.

Theorem 4.19 (Non-stability of strong contraction). There exists a group $G$ with finite generating sets $\mathcal{S} \subset \tilde{\mathcal{S}}$ and an infinite geodesic $\gamma$ in $X:=\operatorname{Cay}(G, \mathcal{S})$ labelled by the powers of a generator such that $\gamma$ is not strongly contracting, but its image $\tilde{\gamma}$ in $\tilde{X}:=\operatorname{Cay}(G, \tilde{\mathcal{S}})$ obtained from the inclusion $\mathcal{S} \subset \tilde{\mathcal{S}}$ is an infinite strongly contracting geodesic.

The idea is to turn [7, Example 3.2] into a $G r^{\prime}(1 / 6)$-labelled graph $\Gamma$. By construction, $\Gamma$ will contain a non-strongly contracting geodesic $\gamma$ that will be labelled by the powers of a generator $y$. Theorem 4.1 then ensures that the image of $\gamma$ in the Cayley graph is not strongly contracting. By adding additional edges, corresponding to new generators, to $\Gamma$ and cutting the resulting graph apart into cycle graphs, we obtain a classical $C^{\prime}(1 / 6)$ presentation of the same group in which no relator contains more than one occurrence of the letter $y$. Thus, a geodesic labelled by the powers of $y$ will be strongly contracting in the Cayley graph with respect to the new generating set.

Proof of Theorem 4.19. Assume the sets $\{y\},\{a, b\},\left\{x_{1}, x_{2}\right\}, \mathcal{S}_{1}, \mathcal{S}_{2}$ are pairwise disjoint sets, and $\left|\mathcal{S}_{1}\right| \geqslant 2$ and $\left|\mathcal{S}_{2}\right| \geqslant 2$. A classical piece with respect to a set of words is the label of a piece in the disjoint union of cycle graphs labelled by the words. It is an exercise to explicitly construct words with the following properties:

Let $\omega:=\left\{w_{1}, w_{2}\right\} \subset\left\langle\mathcal{S}_{1}\right\rangle$ be a $C^{\prime}(1 / 6)$-collection of words (as defined for the proof of Theorem 4.15) such that $\left|w_{1}\right|=\left|w_{2}\right| \geqslant 24$. Moreover, assume that the words $w_{1} w_{2}, w_{1} w_{2}^{-1}$, and $w_{1}^{-1} w_{2}$ are freely reduced.

Let $\mu(a, b):=\left\{\mu_{i}(a, b) \mid i \in \mathbb{N}\right\} \subset\langle\{a, b\}\rangle$ be a $C^{\prime}(1 / 6)$-collection of words such that there exists $C \in \mathbb{N}, C \geqslant 6$ with $\left|\mu_{i}(a, b)\right|=\left(C /\left|w_{1}\right|\right) \cdot 2^{i}$. Note that by our assumptions on $\omega$, the set $\mu\left(w_{1}, w_{2}\right)$ also satisfies the classical $C^{\prime}(1 / 6)$-condition: Since no two $w_{1}^{ \pm 1}$ and $w_{2}^{ \pm 2}$ start with the same letter, any piece with respect to $\mu\left(w_{1}, w_{2}\right)$ comes from a piece with respect to $\mu(a, b)$.

Let $\nu:=\left\{\nu_{i}: i \in \mathbb{N}\right\} \subset\left\langle\mathcal{S}_{2}\right\rangle$ be a $C^{\prime}(1 / 12)$-collection of words such that $\left|\nu_{i}\right|=C \cdot 2^{i}+1$.

The graph $\Gamma$ labelled over $\mathcal{S}:=\{y\} \cup \mathcal{S}_{1} \cup \mathcal{S}_{2}$ is obtained by taking the disjoint union of the $\Gamma_{i}$ as in Figure 14 and, for each $i$, identifying the 'top' of $\Gamma_{i}$ with the 'bottom' of $\Gamma_{i+1}$. These both have the same label. Note that any simple closed path $\gamma$ in $\Gamma$ is a path going around a finite union $\Gamma_{i} \cup \Gamma_{i+1} \cup \cdots \cup \Gamma_{j}$ for $i \leqslant j$, i.e., the label of $\gamma$ is, up to inversion and cyclic shift, of the form:

$$
\mu_{i}\left(w_{1}, w_{2}\right) \nu_{i} \nu_{i+1} \ldots \nu_{j} \mu_{j+1}\left(w_{1}, w_{2}\right)^{-1} y^{i-j-1}
$$


A piece that is a simple subpath of a cyclic shift of $\gamma$ has a label that is a subword of one of the following words.

- A piece in some $\mu_{k}\left(w_{1}, w_{2}\right)$ or a product of two pieces in some $\nu_{k}$ or $\nu_{k} \nu_{k+1}$ (since, by gluing together words $\nu_{k}$ when constructing $\Gamma$, new pieces may have arisen, each labelled by a product of two pieces in $\nu$ ), or

- $y^{i-j-1}$, or

- a product of two words from the first bullet, or a product $p y^{i-j-1} q$, where $p$ and $q$ are pieces in $\mu_{j+1}\left(w_{1}, w_{2}\right)$ and $\mu_{i}\left(w_{1}, w_{2}\right)$ respectively.

Here, a piece in $\mu_{i}\left(w_{1}, w_{2}\right)$ means a classical piece with respect to the collection of words $\mu\left(w_{1}, w_{2}\right)$, a piece in $\nu_{i}$ means a piece with respect to $\nu$, and so on. A piece may also be the empty word.

Note that any piece $p$ in the first bullet has length less than $1 / 6$ of its ambient word $\mu_{k}\left(x_{1}, x_{2}\right)$ or $\nu_{k}$ or $\nu_{k} \nu_{k+1}$, and that $6|i-j-1| \leqslant C 2^{j}<\left|\nu_{j}\right|$. Therefore, $\Gamma$ satisfies the $G r^{\prime}(1 / 6)$-condition.

Let $\alpha$ be the geodesic ray in $\Gamma$ labelled by positive powers of $y$. Let $\beta$ be the geodesic ray in $\Gamma$ labelled by $\nu_{1} \nu_{2} \cdots$. The ray $\beta$ leaves every bounded neighborhood of $\alpha$, but has unbounded image under the closest point projection to $\alpha$ in $\Gamma$. By Corollary 4.5, $\alpha$ is not strongly contracting in $\Gamma$. Thus, by Theorem 4.1, the geodesic ray labelled by positive powers of $y$ is not strongly contracting in $\operatorname{Cay}(G(\Gamma), \mathcal{S})$.

We now define a graph $\tilde{\Gamma}$ labelled over $\tilde{\mathcal{S}}:=\{y\} \cup\left\{x_{1}, x_{2}\right\} \cup \mathcal{S}_{1} \cup \mathcal{S}_{2}$ as follows: Let $c_{1}$ be a cycle graph labelled by $x_{1} w_{1}^{-1}$ and $c_{2}$ a cycle graph labelled by $x_{2} w_{2}^{-1}$. Set:

$$
\tilde{\Gamma}:=c_{1} \sqcup c_{2} \sqcup \bigsqcup_{i \in \mathbb{N}} \tilde{\Gamma}_{i}
$$

where the $\tilde{\Gamma}_{i}$ are given in Figure 14. Note that while, in this new graph, the paths labelled by $\mu_{i}\left(x_{1}, x_{2}\right)$ are pieces, no path labelled by $\mu_{i+1}^{-1}\left(x_{1}, x_{2}\right) y^{-1}$ or $y^{-1} \mu_{i}\left(x_{1}, x_{2}\right)$ is a piece.

By construction, $\left|\mu_{i}\left(x_{1}, x_{2}\right)\right|,\left|\mu_{i+1}\left(x_{1}, x_{2}\right)\right|<\left|\nu_{i}\right| / 12$, and any piece in $\nu_{i}$ has length less than $\left|\nu_{i}\right| / 12$. This, together with observations as above and the fact that any simple piece in $c_{1}$ or $c_{2}$ has length less than $\left|c_{i}\right| / 6$ by construction, shows that $\tilde{\Gamma}$ satisfies the $G r^{\prime}(1 / 6)-$ condition. Since its components are cycle graphs, the geodesic ray labelled by the positive powers of $y$ is strongly contracting in $\operatorname{Cay}(G(\tilde{\Gamma}), \tilde{\mathcal{S}})$, by Theorem 4.1 .

The presentation for $G(\tilde{\Gamma})$ coming from $\tilde{\Gamma}$ includes the generators and relations of the presentation of $G(\Gamma)$ coming from $G(\Gamma)$, as well as generators $x_{1}$ and $x_{2}$ and relations $x_{1} w_{1}^{-1}$ and $x_{2} w_{2}^{-1}$. Rewriting the presentation by Tietze transformations, we see $G(\Gamma) \cong G(\tilde{\Gamma})$. In particular, the inclusion $\operatorname{Cay}(G(\Gamma), \mathcal{S}) \hookrightarrow \operatorname{Cay}(G(\tilde{\Gamma}), \tilde{\mathcal{S}})$ is a quasi-isometry.

We record the following consequence of Theorem 4.1 and Lemma 4.3 for reference:

Corollary 4.20. Let $\alpha$ and $\alpha^{\prime}$ be infinite geodesic rays in $\operatorname{Cay}(G(\Gamma), \mathcal{S})$, where $\Gamma$ is a $G r^{\prime}(1 / 6)$-labelled graph labelled by $\mathcal{S}$, such that $d_{\text {Hausdorff }}\left(\alpha, \alpha^{\prime}\right)<\infty$. Then $\alpha$ is uniformly locally strongly contracting if and only if $\alpha^{\prime}$ is.

\section{Strongly CONTRACTing Elements}

In this section, we show the existence of strongly contracting elements in graphical small cancellation groups.

Theorem 5.1. Let $\Gamma$ be a $G r^{\prime}(1 / 6)$-labelled graph whose components are finite, labelled by a finite set $\mathcal{S}$. Assume that $G(\Gamma)$ is infinite. Then there exists an infinite order element $g \in G(\Gamma)$ such that $\langle g\rangle$ is strongly contracting in $\operatorname{Cay}(G(\Gamma), \mathcal{S})$. 
The element $g$ is the WPD element for the action of $G(\Gamma)$ on the hyperbolic coned-off space of Gruber and Sisto [31] (see also Section 5.2).

A recent theorem of Arzhantseva, Cashen, and Tao [8] says that if $G$ is a group acting cocompactly on a proper metric space $X$, and if $g \in G$ is an infinite order element such that closest point projection to an orbit of $\langle g\rangle$ is strongly contracting, then the action of $G$ on $X$ is growth tight. This means that the rate of exponential growth of $G$ with respect to the pseudo-metric induced by the metric of $X$ is strictly greater than the growth rate of a quotient of $G$ by any infinite normal subgroup, with respect to the induced pseudo-metric on the quotient group. Thus, a corollary of Theorem 5.1, is:

Theorem 5.2. Let $\Gamma$ be a $G r^{\prime}(1 / 6)$-labelled graph whose components are finite, labelled by a finite set $\mathcal{S}$. Then the action of $G(\Gamma)$ on $\operatorname{Cay}(G(\Gamma), \mathcal{S})$ is growth tight.

5.1. Infinite cyclic subgroups are close to periodic geodesics. In the previous section we deduced contraction results for geodesics in a group defined by a $G r^{\prime}(1 / 6)$ labelled graph. In order to show that cyclic subgroups are strongly contracting, we show that they are actually close to bi-infinite geodesics. As a by-product, we also obtain a result about translation lengths in graphical small cancellation groups.

We glean from [28] the following:

Lemma 5.3. Let $\Gamma$ be a $G r^{\prime}(1 / 6)$-labelled graph whose components are finite, labelled by a finite set $\mathcal{S}$. Every infinite cyclic subgroup of $G:=G(\Gamma)$ is at bounded Hausdorff distance from a periodic bi-infinite geodesic in $\operatorname{Cay}(G, \mathcal{S})$.

Recall that a bi-infinite path graph $\alpha$ in $\operatorname{Cay}(G, \mathcal{S})$ is periodic if there is a cyclic subgroup of $G$ that stabilizes $\alpha$ and acts cocompactly on it. In the proof we have surjections $\langle\mathcal{S}\rangle \rightarrow H \rightarrow G$. Let $|\cdot|_{H}$ denote the word length in $H$ with respect to the image of $\mathcal{S}$. Similarly, let $|\cdot|_{G}$ denote the word length in $G$ with respect to the image of $\mathcal{S}$.

Recall that the translation length of an element $g \in G$ is defined by:

$$
\tau_{G}(g):=\lim _{n \rightarrow \infty} \frac{\left|g^{n}\right|_{G}}{n}
$$

This limit always exists since the map $n \mapsto\left|g^{n}\right|_{G}$ is subadditive.

Proof of Lemma 5.3. Let $x$ be an infinite order element of $G$.

Claim. There exist $w \in\langle\mathcal{S}\rangle$ and $N \in \mathbb{N}$ such that $w$ represents an element $g \in G$ such that $x$ is conjugate to $g^{N}$ and the following property is satisfied: There exist a hyperbolic group $H$, also a quotient of $\langle\mathcal{S}\rangle$, and an epimorphism $\phi: H \rightarrow G$ induced by the identity on $\mathcal{S}$ such that, if $h$ denotes the element of $H$ represented by $w$, then $\phi$ restricts to an isometry $\langle h\rangle \rightarrow\langle g\rangle$ with respect to the subspace metrics in $H$ and $G$, respectively.

We show how to deduce the statement of the lemma from the claim: By a theorem of Swenson [50, Theorem 8], since $H$ is hyperbolic, there exist $h_{0} \in H$ and $M \in \mathbb{N}$ such that $h^{M}$ is conjugate to $h_{0}$ and such that $\left|h_{0}^{n}\right|_{H}=n\left|h_{0}\right|_{H}$ for all $n>0$. Therefore, $\tau_{H}\left(h^{M}\right)=\left|h_{0}\right|_{H}$, and the bi-infinite path graph in $H$ labelled by the powers of a shortest element $w_{0} \in\langle\mathcal{S}\rangle$ representing $h_{0}$ is geodesic. Consider $g_{0}:=\phi\left(h_{0}\right)$, the element of $G$ represented by $w_{0}$. Then $g^{M}$ is conjugate to $g_{0}$ by construction, and we have $\tau_{G}\left(g_{0}\right)=$ $\tau_{G}\left(g^{M}\right)=\tau_{H}\left(h^{M}\right)=\left|w_{0}\right|$ since $\langle h\rangle \rightarrow\langle g\rangle$ is an isometry. This implies $\left|g^{n M}\right|_{G}=n\left|w_{0}\right|$ for every $n$, i.e., the bi-infinite path graph in $\operatorname{Cay}(G, \mathcal{S})$ starting at 1 and labelled by the powers of $w_{0}$ is geodesic. This proves the lemma, assuming our claim.

It remains to show the claim: By [28, Section 4], there exists $g \in G$ such that $x$ is conjugate to $g^{N}$ for some $N$ and such that, if $w$ is a shortest word representing $g$, we have 
the following possibilities: Any bi-infinite periodic path graph $\gamma$ labelled by the powers of $w$ is a convex geodesic (Case 1 in [28, Section 4]), or there exists some $C_{0}$ such that for any copy $c$ in $\operatorname{Cay}(G, \mathcal{S})$ of a simple cycle in $\Gamma$, the length of the intersection of $c$ with $\gamma$ is at most $C_{0}$ (Cases $2 \mathrm{a}$ and $2 \mathrm{~b}$ in [28, Section 4$\left.]\right)$. If the first possibility is true, then the claim holds for $H=\langle\mathcal{S}\rangle$.

Assume there exists a $C_{0}$ as in the second possibility. Then we have the following property by [28, Proof of Theorem 4.2 in Case 2a] and [28, Lemma 4.17]: Whenever $v$ is a geodesic word representing $g^{n}$ for some $n$, then the equation $v=w^{n}$ already holds in $G\left(\Gamma^{<6 C_{0}}\right)$, where $\Gamma^{<6 C_{0}}$ denotes the subgraph of $\Gamma$ that is the union of all components with girth less than $6 C_{0}$. Therefore, the epimorphism $G\left(\Gamma^{<6 C_{0}}\right) \rightarrow G$ induced by the identity on $\mathcal{S}$ restricts to an isometry on the cyclic subgroup generated by the element of $G\left(\Gamma^{<6 C_{0}}\right)$ represented by $w$. The graph $\Gamma^{<6 C_{0}}$ is (up to identifying isomorphic components) a finite $G r^{\prime}(1 / 6)$-labelled graph, so $G\left(\Gamma^{<6 C_{0}}\right)$ is a hyperbolic group.

A result on translation lengths. We record another application of our investigations. We show:

Theorem 5.4. Let $\Gamma$ be a $G r^{\prime}(1 / 6)$-labelled graph whose components are finite, labelled by a finite set $\mathcal{S}$. Then every infinite order element of $G(\Gamma)$ has rational translation length, and translation lengths are bounded away from zero.

Recall that similar theorems are true for hyperbolic groups [25, 50, 20] and for the action of the mapping class group of a surface on its curve complex [15].

The rationality statement is a direct consequence of Lemma 5.3. We prove the remaining statement:

Proposition 5.5. Let $\Gamma$ be a $G r^{\prime}(1 / 6)$-labelled graph whose components are finite, labelled by a finite set $\mathcal{S}$. Every infinite order element $x$ of $G:=G(\Gamma)$ has $\tau_{G}(x) \geqslant 1 / 3$.

Proof. Let $x$ be an infinite order element of $G$. By [28, Section 4], there exists $g \in G$ such that $x$ is conjugate to $g^{N}$ for some $N$ and such that, if $w$ is a shortest word representing $g$, we have the following possibilities: Any bi-infinite periodic path graph $\gamma$ labelled by the powers of $w$ is a convex geodesic (Case 1 in [28, Section 4]), or, for each $n$ and any shortest word $g_{n}$ representing $g^{n}$, there exists a diagram $B_{n}$ over $\Gamma$ whose boundary word is $g_{n} w^{-n}$, such that $B_{n}$ is a combinatorial geodesic bigon with respect to the obvious decomposition of $\partial B_{n}$ (Cases 2a, 2b in [28, Section 4]). In particular, every disk component of $B_{n}$ is a single face, or has shape $\mathrm{I}_{1}$.

If the first possibility is true, then $\tau_{G}(g)=|w| \geqslant 1$. Now consider the second possibility. In Case 2a, it follows from [28, Proof of Theorem 4.2 in Case 2a] that any face $\Pi$ of $B_{n}$, the length of the intersection of $\Pi$ with the side of $B_{n}$ corresponding to $g_{n}$ (the bottom) is more than $1 / 3$ times the length of its intersection with the side corresponding to $w^{n}$ (the top). Therefore, in this case, $\left|g_{n}\right|>n|w| / 3 \geqslant n / 3$. In Case $2 \mathrm{~b}$, if a face $\Pi$ intersects the top in at most $|\partial \Pi| / 2$, then it intersects the bottom in more than $|\partial \Pi| / 6$ by the small cancellation condition. If $\Pi$ intersects the top in more than $|\partial \Pi| / 2$, then [28, Lemma 4.8] implies that the intersection of $\Pi$ with the top has length less than $2|w|$. The intersection with the bottom has length at least 1 , since every disk component of $B_{n}$ has shape $\mathrm{I}_{1}$. Therefore, we have $\left|g_{n}\right|>n|w| \cdot \min \left\{\frac{1}{3}, \frac{1}{2|w|}\right\} \geqslant \frac{n}{3}$. We conclude $\tau_{G}(g) \geqslant 1 / 3$. Hence, $\tau_{G}(x)=\tau_{G}\left(g^{N}\right)=|N| \tau_{G}(g) \geqslant N / 3 \geqslant 1 / 3$.

5.2. The coned-off space. In [31], Gruber and Sisto prove that non-elementary groups defined by $G r(7)$-labelled graphs, which, in particular, includes those defined by $G r^{\prime}(1 / 6)$ labelled graphs, are acylindrically hyperbolic. They prove this result by studying the 
action of $G(\Gamma)$ on what we call the coned-off space $Y$ defined as follows: given a graph $\Gamma$ labelled by $\mathcal{S}$, let $\mathcal{W}$ denote the set of all elements of $G(\Gamma)$ represented by words read on (not necessarily closed!) paths in $\Gamma$. We set $Y:=\operatorname{Cay}(G(\Gamma), \mathcal{S} \cup \mathcal{W})$. Thus, we obtain $Y$ from $X:=\operatorname{Cay}(G(\Gamma), \mathcal{S})$ by attaching to every embedded component of $\Gamma$ in $X$ a complete graph.

The proof in [31] shows hyperbolicity of the space $Y$ and existence of an element of $G(\Gamma)$ whose action on $Y$ is hyperbolic and weakly properly discontinuous (WPD). By a theorem of Osin [41], this yields acylindrical hyperbolicity.

Lemma 5.6. Let $\Gamma$ be a $G r^{\prime}(1 / 6)$-labelled graph. Let $g$ be an infinite-order element of $G:=G(\Gamma)$. Let $X:=\operatorname{Cay}(G, \mathcal{S})$, and let $Y$ be the coned-off space. Let $\gamma$ be a bi-infinite geodesic in $X$ that is at finite Hausdorff distance (in $X)$ from $\langle g\rangle$. Then $g$ is hyperbolic for the action of $G$ on $Y$ if and only if there exists $C>0$ such that for every embedded component $\Gamma_{0}$ of $\Gamma$ in $X$ we have $\operatorname{diam}_{\mathrm{X}}\left(\gamma \cap \Gamma_{0}\right)<C$.

Combining this with Lemma 4.3 and Theorem 4.1, we obtain the following.

Corollary 5.7. If $\langle g\rangle$ is within bounded Hausdorff distance of a bi-infinite geodesic in $X$ and acts hyperbolically on $Y$, then $\langle g\rangle$ is strongly contracting in $X$.

Remark 5.8. The converse of Corollary 5.7 fails. Consider the group defined by $\sqcup_{i \in \mathbb{N}} \Gamma_{i}$ constructed in Example 4.18. The subgroup $\langle a\rangle$ is strongly contracting, but in the coned-off space $Y$ its orbit has diameter at most 2, so $a$ does not act hyperbolically on $Y$. Thus, the methods of [31] do not detect that $\langle a\rangle$ is strongly contracting.

Proof of Lemma 5.6 . Let $\gamma$ be a bi-infinite geodesic in $X$ whose Hausdorff distance from $\langle g\rangle$ is equal to $\epsilon<\infty$.

Suppose for every $n \in \mathbb{N}$ there exists an embedded component $\Gamma_{n}$ of $\Gamma$ in $X$ such that $\operatorname{diam}_{X}\left(\Gamma_{n} \cap \gamma\right) \geqslant n$. Denote by $\gamma_{n}$ the path graph $\Gamma_{n} \cap \gamma$. (This is a connected set since each $\Gamma_{n}$ is convex by [31, Lemma 2.15].) Let $\iota \gamma_{n}$ and $\tau \gamma_{n}$ denote, respectively, the initial and terminal vertices of $\gamma_{n}$. Then, by assumption, for each $n$, there exist $m_{n}$ and $l_{n}$ such that $d_{X}\left(\iota \gamma_{n}, g^{m_{n}}\right)<\epsilon$ and $d_{X}\left(\tau \gamma_{n}, g^{l_{n}}\right)<\epsilon$ and, since $\left|\gamma_{n}\right| \rightarrow \infty$, we have $\left|m_{n}-l_{n}\right| \rightarrow \infty$. We have $d_{Y}\left(g^{m_{n}}, g^{l_{n}}\right)<2 \epsilon+1$, since the vertex set of $\Gamma_{n}$ has diameter at most 1 in the metric of $Y$. Therefore, the map $\mathbb{Z} \rightarrow Y: z \mapsto g^{z}$ is not a quasi-isometric embedding.

On the other hand, suppose there exists $C$ such that, for every embedded component $\Gamma_{0}$ of $\Gamma$, we have $\operatorname{diam}_{X}\left(\gamma \cap \Gamma_{0}\right)<C$. Then, by [31, Proposition 3.6], we have, for any $k$ and $l$, that $d_{Y}\left(g^{k}, g^{l}\right)>\frac{1}{C}\left(d_{X}\left(g^{k}, g^{l}\right)-2 \epsilon\right)-2 \epsilon$. Since $\langle g\rangle$ is undistorted in $G$ by [28, Theorem 4.2], this gives the lower quasi-isometry bound for the map $\mathbb{Z} \rightarrow Y: z \mapsto g^{z}$. Since this map is obviously Lipschitz, it is, in fact, a quasi-isometric embedding, whence $g$ is hyperbolic.

We will use the following result of [31]:

Proposition 5.9 ([31, Section 4]). Let $\Gamma$ be a $G r^{\prime}(1 / 6)$-labelled graph. Suppose that $\Gamma$ has at least two non-isomorphic components that each contain an embedded cycle of length at least 2. Then $G(\Gamma)$ contains a hyperbolic element $g$ for the action of $G(\Gamma)$ on the coned-off space $Y$.

5.3. Proof of Theorem 5.1. If $\Gamma$ has only finitely many pairwise non-isomorphic components with non-trivial fundamental groups, then $G(\Gamma)$ is Gromov hyperbolic $[38,27]$ and, hence, the result holds.

If $\Gamma$ has infinitely many pairwise non-isomorphic components with non-trivial fundamental groups, then, since $\mathcal{S}$ is finite, there exist at least two such components each containing 
an embedded cycle of length at least 2. Therefore, by Proposition 5.9, there exists a hyperbolic element $g$ for the action of $G(\Gamma)$ on $Y$. Since the components of $\Gamma$ are finite, Lemma 5.3 yields that $\langle g\rangle$ is within bounded Hausdorff distance of a bi-infinite geodesic in $X$. Therefore, Corollary 5.7 implies the result.

\section{Hyperbolically embedded SubGroups}

6.1. Contracting subgroups and hyperbolically embedded subgroups. We recall a definition of hyperbolically embedded subgroups from [19].

Definition 6.1 (Hyperbolically embedded subgroup). Let $G$ be a group and $H$ a subgroup. Then $H$ is hyperbolically embedded in $G$ if there exists a subset $\mathcal{S}$ of $G$ with the properties below. We denote by $X^{H}$ the Cayley graph of $H$ with respect to $H$, considered as subgraph of $X:=\operatorname{Cay}(G, H \sqcup \mathcal{S})$, and by $\hat{d}$ the metric on $H$ obtained as follows: we define $\hat{d}(x, y)$ to be the length of a shortest path from $x$ to $y$ in $X$ that does not use any edges of $X^{H}$. If no such path exists, set $\hat{d}(x, y)=\infty$. The required properties are:

- $H \sqcup \mathcal{S}$ generates $G$ (i.e. $X$ is connected).

- $X$ is Gromov hyperbolic.

- The metric $\hat{d}$ is proper on $H$, i.e. $\hat{d}$-balls are finite.

Note that if $s \in H \cap \mathcal{S}$, then it is our convention that $\operatorname{Cay}(G, H \sqcup \mathcal{S})$ will have a double-edge corresponding to $s$ (once considered as element of $H$ and once considered as element of $\mathcal{S}$ ); hence the symbol $\sqcup$ for disjoint union.

One approach to finding hyperbolically embedded subgroups is provided by [13, Theorem H]. A special case of this theorem states that if $G$ is a finitely generated group and a hyperbolic element $g \in G$ has a strongly contracting orbit in a Cayley graph of $G$, then the elementary closure $E(g)$ of $g$ is an infinite, virtually cyclic, hyperbolically embedded subgroup of $G$.

Combining this with Lemma 4.3 and Theorem 4.1, we obtain the following.

Theorem 6.2. Let $G:=G(\Gamma)$ be the group defined by a $G r^{\prime}(1 / 6)$-labelled graph $\Gamma$. Let $g \in G$ be of infinite order, and let $\gamma$ be a bi-infinite geodesic at finite Hausdorff distance from $\langle g\rangle$. If $\gamma$ is uniformly locally strongly contracting, then $\langle g\rangle$ is strongly contracting and, in particular, the elementary closure $E(g)$ of $g$ is a virtually cyclic hyperbolically embedded subgroup.

Remark 6.3. By [13, Theorem H], we can also view Theorem 5.1 as an alternative proof of acylindrical hyperbolicity for the groups considered in that theorem. The initial proof of acylindrical hyperbolicity of these groups in [31] relies on the hyperbolicity of the coned-off space and on showing that the element $g$ satisfies a certain weak proper discontinuity condition (and, in fact, applies to a larger class of groups). Our Theorem 6.2 gives an alternative proof that the element $g$ from [31] gives rise to a hyperbolically embedded virtually cyclic subgroup.

Every hyperbolically embedded subgroup of a finitely generated group is Morse [46], and every element with a strongly contracting orbit has a virtually cyclic hyperbolically embedded elementary closure. In graphical small cancellation groups, Morse and strongly contracting elements have been classified in terms of the defining graph, by our results in Section 4. It is natural to ask whether the collection of cyclic hyperbolically embedded subgroups can be classified in a similar way. We give one negative result in this direction.

Theorem 6.4. Let $\rho_{2}$ be an unbounded sublinear function. There exists a $G r^{\prime}(1 / 6)$-labelled graph $\Gamma$ with set of labels $\mathcal{S}:=\{a, b\}$ whose components are all cycles such that the group 
$G:=G(\Gamma)$ has the following properties: Any virtually cyclic subgroup $E$ of $G$ containing $\langle a\rangle$ is $\left(r, \rho_{2}^{\prime}\right)$-contracting in the Cayley graph $X:=\operatorname{Cay}(G, \mathcal{S})$ for some $\rho_{2}^{\prime} \asymp \rho_{2}$, but $E$ is not hyperbolically embedded in $G$.

Proof. For every $r>0$, choose $N_{r} \geqslant 6$ such that $\rho_{2}\left(\frac{1}{2}(1+r) N_{r}\right) \geqslant r$. This is possible because $\rho_{2}$ is unbounded. For every $r>0$, let $R_{r}:=\left(a^{r} b a^{r} b^{-1}\right)^{N_{r}}$, and consider the graph $\Gamma$ that is the disjoint union of cycles $\gamma_{r}$ labelled by the $R_{r}$. Any reduced path that is a piece in $\gamma_{r}$ has length at most $2 r+1$, which is less than $\left|\gamma_{r}\right| / 6$. Therefore, $\Gamma$ satisfies the $G r^{\prime}(1 / 6)$-condition. Denote by $\alpha$ a bi-infinite path in $X$ labelled by the powers of $a$. For every copy $\gamma$ of a $\gamma_{r}$ embedded in $X$, we have diam $\alpha \cap \gamma<|\gamma| / 6$. Therefore, it is readily seen from considering diagrams of shape $\mathrm{I}_{1}$ that $\alpha$ is a bi-infinite geodesic. Also note that $\gamma$ intersects $\alpha$ in a path of length at most $r$.

Suppose $\gamma$ is a relator intersecting $\alpha$, and $x$ is a point in $\gamma$ with $\delta:=d(x, \alpha)$. If $|\gamma|>4 \delta$, then, for any point $y$ in $\gamma$ with $d(x, y) \leqslant \delta$, we have $\pi(y)=\pi(x)$, and $\pi(x)$ is a singleton, where $\pi$ denotes closest point projection to $\alpha$. Now assume $|\gamma| \leqslant 4 \delta$, and $y \in \gamma$. Then diam $\pi(x) \cup \pi(y) \leqslant \operatorname{diam} \gamma \cap \alpha \leqslant \rho_{2}(\delta)$, by construction, since $\rho_{2}$ is non-decreasing. Therefore, $\alpha$ is locally $\left(r, \rho_{2}\right)$-contracting and, hence, by Theorem 4.1 and Lemma 4.3 there exists $\rho_{2}^{\prime}$ with $\rho_{2}^{\prime} \asymp \rho_{2}$ such that any virtually cyclic subgroup $E$ of $G$ containing $\langle a\rangle$ is $\left(r, \rho_{2}^{\prime}\right)$-contracting.

If $E$ is a hyperbolically embedded subgroup then, by [19, Theorem 5.3], there exists $r>0$ such that the normal closure $N$ of $a^{r}$ is a free group. By construction, the element of $G$ represented by $a^{r} b a^{r} b^{-1}$ is non-trivial and has finite order, whence $N$ is not torsion-free. Therefore, $E$ is not hyperbolically embedded.

Another natural question is the following.

Question 6.5. Let $G$ be a group generated by a finite set $\mathcal{S}$, and suppose $g \in G$ has a $\left(\rho_{1}, \rho_{2}\right)$-contracting orbit in $X:=\operatorname{Cay}(G, \mathcal{S})$ where $\rho_{2}$ is bounded. Is the elementary closure $E(g)$ a hyperbolically embedded virtually cyclic subgroup of $G$ ?

Is the statement true if $G:=G(\Gamma)$ for a $G r^{\prime}(1 / 6)$-labelled graph $\Gamma$ whose components are finite, with finite set of labels $\mathcal{S}$ ?

In the case of classical $C^{\prime}(1 / 6)$-groups, an affirmative answer follows from Theorem 4.16.

6.2. Hyperbolically embedded cycles. Recall that a group has the hyperbolically embedded cycles property (HEC property) if the elementary closure $E(g)$ of every infinite order element $g$ is virtually cyclic and hyperbolically embedded. Hyperbolic groups have this property: $E(g)$ is the stabilizer of a $g$-axis. The HEC property in fact passes to any subgroup of a hyperbolic group $G$ using the action of the subgroup on a Cayley graph of $G$.

It is therefore very natural to ask whether this classifies subgroups of hyperbolic groups. Torsion presents one complication: A free product of infinite torsion groups, or, more generally, a group hyperbolic relative to infinite torsion subgroups, has the HEC property but cannot be a subgroup of a hyperbolic group. We show that even among torsion-free groups there are many examples of groups with the HEC property that are not subgroups of any hyperbolic group.

Theorem 6.6. There exist $2^{\aleph_{0}}$ pairwise non-quasi-isometric finitely generated torsionfree groups in which every non-trivial cyclic subgroup is strongly contracting and which, therefore, have the HEC property.

Since there are only countably many finitely generated subgroups of finitely presented groups, most of the groups of Theorem 6.6 do not occur as subgroups of hyperbolic groups. 
We show in Corollary 6.10 that there are even exotic examples of groups with the HEC property such as the Gromov monster groups.

The theorem is proven by building small cancellation groups in which no power of any element has long intersection with an embedded component of the defining graph. In Theorem 6.7 we give a condition on the labelling that guarantees this property. In Theorem 6.8 we show that this condition can be satisfied. Then we construct specific examples satisfying our condition and apply a version of a construction of Thomas and Velickovic [51], proven in Proposition 6.13, to show that we get uncountably many quasiisometry classes of groups.

A labelling of the edges of an undirected graph is said to be non-repetitive if there does not exist a non-trivial embedded path graph that is labelled by a word of the form $w w$. (Here, the label of a path is just the concatenation of the labels of the edges in the free monoid on the labelling set.) The Thue number of a graph is the minimal cardinality of a labelling set for which the graph admits a non-repetitive labelling.

We define a labelling of a directed graph to be non-repetitive if there does not exist a non-trivial embedded path graph $\gamma=e_{1}, \ldots, e_{2 n}$ such that for all $1 \leqslant i \leqslant n$ the label of the directed edge $e_{i}$ is equal to the label of the directed edge $e_{i+n}$. Note that, given an undirected graph with a non-repetitive labelling, any choice of orientation gives rise to a non-repetitive labelling of the resulting oriented graph.

Theorem 6.7. Let $\mathcal{S}$ be a finite set and let $\Gamma$ be a graph with finite components and a labelling by $\mathcal{S}$ that is both $G r^{\prime}(1 / 6)$ and non-repetitive. Let $G:=G(\Gamma)$. Every infinite cyclic subgroup $H$ of $G$ is strongly contracting in $X:=\operatorname{Cay}(G, \mathcal{S})$. Thus, $G$ has the $H E C$ property.

Proof. By Lemma 5.3, every infinite cyclic subgroup is bounded Hausdorff distance from a periodic geodesic $\alpha$. By Lemma 4.3, it is enough to show $\alpha$ is strongly contracting, so we may assume that $H=\langle h\rangle$ acts cocompactly on $\alpha$, and that $h$ is represented by a cyclically reduced word $v$ whose powers label $\alpha$.

Suppose $\alpha$ intersects some embedded component $\Gamma_{0}$ in more than a single vertex. Since $\alpha$ is geodesic, $\Gamma_{0} \cap \alpha$ is an embedded path graph. Since the labelling of $\Gamma$ is non-repetitive, the label of this path does not contain a subword of the form $w w$. However, the labelling of $\alpha$ is repetitive - it is $\cdots v v \cdots$. Thus, $\left|\Gamma_{0} \cap \alpha\right|<2|v|$. We conclude $\alpha$ is strongly contracting by Theorem 4.1 .

By [13, Theorem H], the elementary closure of $H$ is a virtually cyclic hyperbolically embedded subgroup.

Suppose that $\Gamma$ is a directed graph with two labellings $L_{1}: \mathcal{E} \Gamma \rightarrow \mathcal{S}_{1}$ and $L_{2}: \mathcal{E} \Gamma \rightarrow \mathcal{S}_{2}$. We define the push-out labelling $L: \mathcal{E} \Gamma \rightarrow \mathcal{S}$, where $\mathcal{S}=\mathcal{S}_{1} \times \mathcal{S}_{2}$, by $L(e):=\left(L_{1}(e), L_{2}(e)\right)$. We will write $\mathcal{S}_{1}$ alphabetically and $\mathcal{S}_{2}$ as numerical index, i.e. $\mathcal{S}^{ \pm}=\left\{a_{n}^{\epsilon} \mid \epsilon \in \pm 1, a \in\right.$ $\left.\mathcal{S}_{1}, n \in \mathcal{S}_{2}\right\}$.

If $L_{2}: \mathcal{E} \Gamma \rightarrow \mathcal{S}_{2}$ is a non-repetitive labelling of $\Gamma$, then for any labelling $L_{1}: \mathcal{E} \Gamma \rightarrow \mathcal{S}_{1}$ the push-out labelling is a non-repetitive labelling of $\Gamma$. Similarly, if $L_{1}: \mathcal{E} \Gamma \rightarrow \mathcal{S}_{1}$ satisfies the $C^{\prime}(1 / 6)$-condition, then so does the push-out labelling.

Theorem 6.8. Let $\Gamma=\left(\Gamma_{i}\right)_{i \in \mathbb{N}}$ be a sequence of finite, connected graphs satisfying the following conditions:

- $\Gamma$ has bounded valence.

- $\left(\operatorname{girth}\left(\Gamma_{i}\right)\right)_{i \in \mathbb{N}}$ is an unbounded sequence.

- The ratios $\frac{\operatorname{girth}\left(\Gamma_{i}\right)}{\operatorname{diam}\left(\Gamma_{i}\right)}$ are bounded, uniformly over $i$, away from 0. 
Then there exist an infinite subsequence $\left(\Gamma_{i_{j}}\right)_{j \in \mathbb{N}}$ of graphs and a finite set $\mathcal{S}$ such that $\left(\Gamma_{i_{j}}\right)_{j \in \mathbb{N}}$ admits an labelling by $\mathcal{S}$ that is both $C^{\prime}(1 / 6)$ and non-repetitive.

Proof. A theorem of Osajda [40] says that, given the hypotheses on $\Gamma$, there exists an infinite subsequence $\left(\Gamma_{i_{j}}\right)_{j \in \mathbb{N}}$ that admits a choice of orientation and a labelling by a finite set $\mathcal{S}_{1}$ satisfying the $C^{\prime}(1 / 6)$-condition. Alon, et al. [3] show that the Thue number of a bounded valence undirected graph is bounded by a polynomial function of the valence bound. Since $\Gamma$ has bounded valence, there exists a finite set of labels $\mathcal{S}_{2}$ such that $\Gamma$ admits a non-repetitive $\mathcal{S}_{2}$-labelling (as undirected graph and, hence, also as directed graph). The push-out of these two labellings satisfies the theorem.

Combining this with Theorem 6.7, we have:

Corollary 6.9. If $\Gamma$ is as in Theorem 6.8 then $G(\Gamma)$ has the HEC property.

Corollary 6.10. There exist Gromov monster groups with the HEC property.

More generally, if $\Gamma$ is a bounded valence graph with a labelling satisfying some property $\mathcal{P}$ such that $\mathcal{P}$ is preserved upon passing to a refinement of the labelling, then $\Gamma$ admits a labelling that is both $\mathcal{P}$ and non-repetitive. For instance, Arzhantseva and Osajda [4] introduced a 'lacunary walling condition' to produce first examples of non-coarsely amenable groups ${ }^{2}$ with the Haagerup property. This condition is preserved upon passing to refinements of the labelling, so the same argument as in Theorem 6.8 yields:

Corollary 6.11. There exist finitely generated, non-coarsely amenable groups with the Haagerup property and the HEC property.

Not every interesting property of labellings is preserved upon passing to refinements. For example, the $G r^{\prime}(\lambda)$ condition is not preserved, because in the original labelling there may be a long labelled path $p$ with distinct label-preserving maps $\phi_{1}, \phi_{2}: p \rightarrow \Gamma$ and a label-preserving automorphism $\psi$ of $\Gamma$ such that $\phi_{2}=\psi \circ \phi_{1}$. If $\psi$ fails to be a label-preserving automorphism of $\Gamma$ with the refined labelling then $p$ may be too long a piece.

For another example, passing to a refinement can yield a group with non-trivial free factors. In particular, no property of a labelling that implies the resulting group has Kazhdan's Property (T) is preserved by passing to refinements.

Both of the factor labellings in the proof of Theorem 6.8 are produced probabilistically using the Lovász Local Lemma. For the purpose of proving Theorem 6.6 we construct explicit examples of labelled graphs satisfying Theorem 6.7, so we get concrete examples of groups satisfying Theorem 6.6. To construct such examples we use the fact, first observed by Alon, et al. [3], that a cycle graph has Thue number at most 4, as follows:

Definition 6.12 (Thue-Morse sequence). Define $\sigma:\{0,1\}^{*} \rightarrow\{0,1\}^{*}$ by $\sigma(0):=01$, $\sigma(1):=10$. The sequence $\left(x_{i}\right)_{i \in \mathbb{N}}:=\sigma^{\infty}(0)$ is called the Thue-Morse sequence ${ }^{3}$.

The Thue-Morse sequence famously does not contain any subword of the form $w w w$. The 'first difference' sequence ${ }^{4}\left(y_{i}\right)_{i \in \mathbb{N}} \in\{-1,0,1\}^{\mathbb{N}}$ defined by $y_{i}:=x_{i+1}-x_{i}$, where $\left(x_{i}\right)_{i \in \mathbb{N}}$ is the Thue-Morse sequence, does not contain any subword of the form $w w$, which means that it gives a non-repetitive labelling of the ray graph. Both of these facts are due to Thue [52].

\footnotetext{
${ }^{2}$ These are groups $G$ whose reduced $C^{*}$-algebra $C_{r e d}^{*}(G)$ is not exact.

${ }^{3}$ Sequence A010060 of [1].

${ }^{4}$ Sequence A029883 of [1].
} 
A cycle graph of length $n$ with edges $e_{0}, e_{1}, \ldots, e_{n-1}$ admits a non-repetitive labelling by $\{-1,0,1, \infty\}$ by labelling edge $e_{0}$ with $\infty$ and labelling edge $e_{i}$ for $i>0$ with term $y_{i}$ of the first difference of the Thue-Morse sequence.

Define $\Gamma:=\left(\Gamma_{n}\right)_{n \in \mathbb{N}}$ to be a disjoint union of cycle graphs such that $\left|\Gamma_{n}\right|=11(44 n-19)$. Give each of these cycles the non-repetitive $\{-1,0,1, \infty\}$-labelling defined above. Also give $\Gamma_{n}$ the $\{a, b\}$-labelling $R_{n}:=\prod_{i=22 n-21}^{22 n} a b^{i}$. This is a $C^{\prime}(1 / 6)$-labelling. There are no label-preserving automorphisms of $\Gamma$ since the components are cycles of different lengths and are labelled by positive words that are not proper powers. If $p$ is a piece contained in $\Gamma_{n}$ then, since the gaps between $a$ 's in the $R_{i}$ are all different, $p$ contains at most one edge labelled $a$. The longest subword of $R_{n}$ containing at most one $a$ is $b^{22 n-1} a b^{22 n}$ of length $44 n$. For $n \in \mathbb{N}$, the ratio $\frac{44 n}{11(44 n-19)}$ takes maximum value $4 / 25<1 / 6$ at $n=1$.

The push-out of these two labellings is an $\mathcal{S}$-labelling of $\Gamma$ that is both non-repetitive and $C^{\prime}(1 / 6)$, with $\mathcal{S}:=\left\{a_{-1}, a_{0}, a_{1}, a_{\infty}, b_{-1}, b_{0}, b_{1}, b_{\infty}\right\}$.

For each subset $I \subset \mathbb{N}$ define $\Gamma_{I}:=\left(\Gamma_{n}\right)_{n \in I}$. All of these graphs have non-repetitive, $C^{\prime}(1 / 6)$-labellings inherited from $\Gamma$. By Theorem 6.7, every non-trivial cyclic subgroup of the group $G_{I}$ defined by $\Gamma_{I}$ is strongly contracting. The proof of Theorem 6.6 is completed by the following proposition and the fact that groups defined by $C^{\prime}(1 / 6)$-labelled graphs are torsion-free [27].

Proposition 6.13 ([51]). There is a subset $\mathcal{I} \subseteq 2^{\mathbb{N}}$ of cardinality $2^{\aleph_{0}}$ such that given $I, J \in \mathcal{I}$, the groups $G_{I}$ and $G_{J}$ are quasi-isometric if and only if $I=J$.

Proof. Choose $\mathcal{I}$ to be a collection of infinite subsets of $\left\{2^{2^{n}} \mid n \in \mathbb{N}\right\}$ with infinite pairwise symmetric difference.

Let $I, J \in \mathcal{I}$ be distinct. Without loss of generality, assume $I \backslash J$ is infinite, and let $\mu$ be a non-atomic ultrafilter on $\mathbb{N}$ with $\mu(I)=1$ and $\mu(J)=0$.

The asymptotic cone of $G_{J}$ over $\mu$ with scaling sequence $\left(\left|R_{n}\right|\right)_{n \in I}$ is an $\mathbb{R}$-tree, while the asymptotic cone of $G_{I}$ over $\mu$ with the same scaling contains a loop of length 1 .

If $G_{I}$ and $G_{J}$ are quasi-isometric then their asymptotic cones are bi-Lipschitz equivalent. Thus these groups are not quasi-isometric.

\section{REFERENCES}

[1] The on-line encyclopedia of integer sequences, http://oeis.org.

[2] Yael Algom-Kfir, Strongly contracting geodesics in outer space, Geom. Topol. 15 (2011), no. 4, 2181-2233.

[3] Noga Alon, Jarosław Grytczuk, Mariusz Hałuszczak, and Oliver Riordan, Nonrepetitive colorings of graphs, Random Structures \& Algorithms 21 (2002), no. 3-4, 336-346.

[4] Goulnara Arzhantseva and Damian Osajda, Graphical small cancellation groups with the Haagerup property, preprint (2014), arXiv: 1404.6807v2.

[5] Goulnara Arzhantseva and Markus Steenbock, Rips construction without unique product, preprint (2014), arXiv: 1407.2441.

[6] Goulnara Arzhantseva and Romain Tessera, Admitting a coarse embedding is not preserved under group extensions, Int. Math. Res. Not. IMRN (in press).

[7] Goulnara N. Arzhantseva, Christopher H. Cashen, Dominik Gruber, and David Hume, Characterizations of Morse geodesics via superlinear divergence and sublinear contraction, Doc. Math. 22 (2017), 1193-1224.

[8] Goulnara N. Arzhantseva, Christopher H. Cashen, and Jing Tao, Growth tight actions, Pacific J. Math. 278 (2015), no. 1, 1-49.

[9] Goulnara N. Arzhantseva and Thomas Delzant, Examples of random groups, preprint, available on the authors' webpages (2008).

[10] Jason Behrstock, Cornelia Druţu, and Lee Mosher, Thick metric spaces, relative hyperbolicity, and quasi-isometric rigidity, Math. Ann. 344 (2009), no. 3, 543-595. 
[11] Jason Behrstock and Cornelia Druţu, Divergence, thick groups, and short conjugators, Illinois J. Math. 58 (2014), no. 4, 939-980.

[12] Jason A. Behrstock, Asymptotic geometry of the mapping class group and Teichmüller space, Geom. Topol. 10 (2006), 1523-1578.

[13] Mladen Bestvina, Kenneth Bromberg, and Koji Fujiwara, Constructing group actions on quasi-trees and applications to mapping class groups, Publ. Math. Inst. Hautes Études Sci. 122 (2015), no. 1, $1-64$.

[14] Mladen Bestvina and Koji Fujiwara, A characterization of higher rank symmetric spaces via bounded cohomology, Geom. Funct. Anal. 19 (2009), no. 1, 11-40.

[15] Brian H. Bowditch, Tight geodesics in the curve complex, Invent. Math. 171 (2008), no. 2, 281-300.

[16] Gregory R. Conner, A class of finitely generated groups with irrational translation numbers, Arch. Math. (Basel) 69 (1997), no. 4, 265-274.

[17] Gregory R. Conner, Translation numbers of groups acting on quasiconvex spaces, Computational and geometric aspects of modern algebra (Edinburgh, 1998), London Math. Soc. Lecture Note Ser., vol. 275, Cambridge Univ. Press, Cambridge, 2000, pp. 28-38.

[18] Rémi Cunéo and Hamish Short, Graph small cancellation theory applied to alternating link groups, Journal of Knot Theory and Its Ramifications 21 (2012), no. 11, 1250113, 15 pages.

[19] François Dahmani, Vincent Guirardel, and Denis Osin, Hyperbolically embedded subgroups and rotating families in groups acting on hyperbolic spaces, Mem. Amer. Math. Soc. 245 (2017), no. 1156, v+152.

[20] Thomas Delzant, Sous-groupes distingués et quotients des groupes hyperboliques, Duke Math. J. 83 (1996), no. 3, 661-682.

[21] Cornelia Druţu, Shahar Mozes, and Mark Sapir, Divergence in lattices in semisimple Lie groups and graphs of groups, Trans. Amer. Math. Soc. 362 (2010), no. 5, 2451-2505.

[22] Cornelia Druţu and Mark Sapir, Tree-graded spaces and asymptotic cones of groups, Topology 44 (2005), no. 5, 959-1058, With an appendix by D. Osin and Sapir.

[23] Moon Duchin and Kasra Rafi, Divergence of geodesics in Teichmüller space and the mapping class group, Geom. Funct. Anal. 19 (2009), no. 3, 722-742.

[24] Rostislav Grigorchuk and Pierre de la Harpe, On problems related to growth, entropy, and spectrum in group theory, J. Dynam. Control Systems 3 (1997), no. 1, 51-89.

[25] Mikhael Gromov, Hyperbolic groups, Essays in group theory, Math. Sci. Res. Inst. Publ., vol. 8, Springer, New York, 1987, pp. 75-263.

[26] Mikhael Gromov, Random walk in random groups, Geom. Funct. Anal. 13 (2003), no. 1, 73-146.

[27] Dominik Gruber, Groups with graphical $C(6)$ and $C(7)$ small cancellation presentations, Trans. Amer. Math. Soc. 367 (2015), no. 3, 2051-2078.

[28] Dominik Gruber, Infinitely presented C(6)-groups are SQ-universal, J. Lond. Math. Soc. 92 (2015), no. $1,178-201$.

[29] Dominik Gruber, Infinitely presented graphical small cancellation groups: Coarse embeddings, acylindrical hyperbolicity, and subgroup constructions, Ph.D. thesis, University of Vienna, 2015.

[30] Dominik Gruber, Alexandre Martin, and Markus Steenbock, Finite index subgroups without unique product in graphical small cancellation groups, Bull. London Math. Soc. 47 (2015), no. 4, 631-638.

[31] Dominik Gruber and Alessandro Sisto, Infinitely presented graphical small cancellation groups are acylindrically hyperbolic, Ann. Inst. Fourier (in press).

[32] Nigel Higson, Vincent Lafforgue, and George Skandalis, Counterexamples to the Baum-Connes conjecture, Geom. Funct. Anal. 12 (2002), no. 2, 330-354.

[33] David Hume, A continuum of expanders, Fund. Math. 238 (2017), no. 2, 143-152.

[34] Ilya Kapovich, Small cancellation groups and translation numbers, Trans. Amer. Math. Soc. 349 (1997), no. 5, 1851-1875.

[35] Howard A. Masur and Yair N. Minsky, Geometry of the complex of curves. I. Hyperbolicity, Invent. Math. 138 (1999), no. 1, 103-149.

[36] Howard A. Masur and Yair N. Minsky, Geometry of the complex of curves. II. Hierarchical structure, Geom. Funct. Anal. 10 (2000), no. 4, 902-974.

[37] Yair N. Minsky, Quasi-projections in Teichmüller space, J. Reine Angew. Math. 473 (1996), 121-136.

[38] Yann Ollivier, On a small cancellation theorem of Gromov, Bull. Belg. Math. Soc. Simon Stevin 13 (2006), no. 1, 75-89.

[39] Yann Ollivier and Daniel T. Wise, Kazhdan groups with infinite outer automorphism group, Trans. Amer. Math. Soc. 359 (2007), no. 5, 1959-1976. 
[40] Damian Osajda, Small cancellation labellings of some infinite graphs and applications, preprint (2014), arXiv: $1406.5015 \mathrm{v} 1$.

[41] Denis V. Osin, Acylindrically hyperbolic groups, Trans. Amer. Math. Soc. 368 (2016), no. 2, 851-888.

[42] Stephen Pride, Some problems in combinatorial group theory, Groups-Korea 1988 (Pusan, 1988), Lecture Notes in Math., vol. 1398, Springer, Berlin, 1989, pp. 146-155, http://dx.doi.org/10.1007/BFb0086251.

[43] Eliyahu Rips and Yoav Segev, Torsion-free group without unique product property, J. Algebra 108 (1987), no. 1, 116-126.

[44] Lior Silberman, Addendum to: "Random walk in random groups" [Geom. Funct. Anal. 13 (2003), no. 1, 73-146; mr1978492] by M. Gromov, Geom. Funct. Anal. 13 (2003), no. 1, 147-177.

[45] Alessandro Sisto, Projections and relative hyperbolicity, Enseign. Math. (2) 59 (2013), no. 1-2, $165-181$.

[46] Alessandro Sisto, Quasi-convexity of hyperbolically embedded subgroups, Math. Z. 283 (2016), no. 3-4, 649-658.

[47] Markus Steenbock, Rips-Segev torsion-free groups without the unique product property, J. Algebra 438 (2015), 337-378.

[48] Ralph Strebel, Appendix. Small cancellation groups, Sur les groupes hyperboliques d'après Mikhael Gromov (Bern, 1988), Progr. Math., vol. 83, Birkhäuser Boston, Boston, MA, 1990, pp. 227-273.

[49] Harold Sultan, Hyperbolic quasi-geodesics in CAT(0) spaces, Geom. Dedicata 169 (2014), no. 1, 209-224.

[50] Eric L. Swenson, Hyperbolic elements in negatively curved groups, Geom. Dedicata 55 (1995), no. 2, 199-210.

[51] Simon Thomas and Boban Velickovic, Asymptotic cones of finitely generated groups, Bull. London Math. Soc. 32 (2000), no. 2, 203-208.

[52] Axel Thue, Über die gegenseitige Lage gleicher Teile gewisser Zeichenreihen., Kristiania: J. Dybwad. 67 S. Lex. $8^{\circ}, 1912$.

E-mail address: goulnara.arzhantseva@univie.ac.at

Fakultät für Mathematik, Universität Wien, Oskar-Morgenstern-Platz 1, 1090 Wien, ÖSTERREICH

E-mail address: christopher.cashen@univie.ac.at

Fakultät für Mathematik, Universität Wien, Oskar-Morgenstern-Platz 1, 1090 Wien, ÖSTERREICH

E-mail address: dominik.gruber@math.ethz.ch

Department of Mathematics, ETH Zürich, RÄmistrasse 101, 8092 Zürich, Switzerland

E-mail address: david.hume@maths.ox.ac.uk

Mathematical Institute, University of Oxford, Woodstock Road, OX2 6GG Oxford, UK 